\title{
Analysis of a Full Space-Time Discretization of the Navier-Stokes Equations by a Local Projection Stabilization Method
}

\author{
Naveed Ahmed * Tomás Chacón Rebollo † Volker John ${ }^{\ddagger}$ Samuele Rubino ${ }^{\S}$
}

September 6, 2016

\begin{abstract}
A finite element error analysis of a local projection stabilization (LPS) method for the time-dependent Navier-Stokes equations is presented. The focus is on the high-order term-by-term stabilization method that has one level, in the sense that it is defined on a single mesh, and in which the projection-stabilized structure of standard LPS methods is replaced by an interpolation-stabilized structure. The main contribution is on proving, theoretically and numerically, the optimal convergence order of the arising fully discrete scheme. In addition, the asymptotic energy balance is obtained for slightly smooth flows. Numerical studies support the analytical results and illustrate the potential of the method for the simulation of turbulent flows. Smooth unsteady flows are simulated with optimal order of accuracy.
\end{abstract}

Keywords: evolutionary incompressible Navier-Stokes equations, high order term-byterm LPS scheme, finite element error analysis, high Reynolds number flows

2010 Mathematics Subject Classification: 65M12, 65M60, 76D05.

\section{Introduction}

In the present paper, we address the numerical analysis of fully discrete schemes arising from Finite Element (FE) Local Projection Stabilization (LPS) methods that approximate the unsteady Navier-Stokes Equations (NSE). We mainly focus here on the high-order term-by-term stabilization method (cf. [16]). This method is a particular type of LPS scheme, which constitutes a low-cost, accurate solver for incompressible flows, despite being only weakly consistent. It differs from the standard LPS methods (cf. [10, 39]) because it uses continuous buffer functions, it does not need enriched FE spaces, it does not need element-wise projections satisfying suitable orthogonality properties, and it does not need multiple meshes. An interpolant-stabilized structure replaces the projectionstabilized structure of standard LPS methods. The interpolation operator takes its values in a continuous buffer space, different from the discrete velocity space, but defined on the

\footnotetext{
*WIAS, Mohrenstr. 39, 10117 Berlin, Germany ahmed@wias-berlin.de

${ }^{\dagger}$ Department EDAN \& IMUS, University of Seville, C/Tarfia s/n., 41012 Seville, Spain chacon@us .es

${ }^{\ddagger}$ WIAS, Mohrenstr. 39, 10117 Berlin, and Free University of Berlin, Department of Mathematics and Computer Science, Arnimallee 6, 14195 Berlin, Germany john@wias-berlin.de

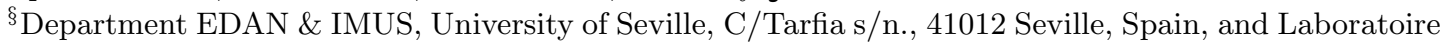
Jacques-Louis Lions, Sorbonne Universités, UPMC Univ. Paris 6, 75005 Paris, France samuele@us.es
} 
same mesh, constituted by standard polynomials with one degree less than the FE space for the velocity. This approach gives rise to a method with reduced computational cost for some choices of the interpolation operator.

LPS schemes were originally proposed for the Stokes problem [6], and then successfully extended to transport problems ( $c f .[2,5,7,38,42,48])$. As classical stabilization procedures, these discretizations are based upon an "augmented" variational formulation of the flow equations, which includes additional terms to the standard Galerkin discretization. They allow to circumvent the discrete inf-sup condition and to use equal order interpolation for velocity and pressure and they also provide stabilization of convection-dominant effects. Different variants of LPS methods have been investigated during the recent years for incompressible flow problems. The main common feature is that the stabilization terms only act on the small scales of the flow, thus ensuring a higher accuracy with respect to more classical stabilization procedures, such as penalty-stabilized methods (cf. [15]). For a detailed description of different variants of LPS schemes, we refer to [32, 39, 51].

The main contribution of this work is to prove, for the proposed method, the optimal convergence accuracy of the arising fully (space-time) discrete scheme (semi-implicit in time), which, to the best of our knowledge, cannot be found in the literature so far.

LPS methods are well-understood for the Oseen problem (cf. [10, 11, 26, 41, 43]). Recent attempts for the extension of the numerical analysis of LPS methods to the time-dependent incompressible NSE can be found in [3, 14], and also in [17] for the LPS method analyzed in the present paper. However, the analysis in [17] proves only stability and weak convergence of the proposed method in natural norms, while in [3, 14] just the space semi-discrete problem is considered. Thus, the present paper aims to complement the available results with the extension of the error analysis to the fully discrete incompressible evolution NSE. Since LPS methods may be cast in the Variational Multi-Scale (VMS) framework ( $c f$. [10]), the present paper also constitutes a step forward to the survey and classification of VMS methods (see [1] for a recent detailed review of VMS methods for the simulation of turbulent incompressible flows). The connection to VMS methods was a motivation to perform the studies presented in this paper.

In this paper, optimal error estimates for smooth unsteady solutions are proved on the basis of specific inf-sup conditions. The error analysis permits to show the strong convergence of the proposed method for slightly smooth flows (i.e., $(\boldsymbol{u}, p) \in C^{0}\left(\mathbf{H}^{2}\right) \times C^{0}\left(H^{1}\right)$ at least) and a subsequent asymptotic energy balance of the system. The convergence order decreases with the regularity of the flow, but potentially maintaining these schemes as suitable and useful tools for the simulation of turbulent flows. We also include numerical tests for the 3D Beltrami flow in laminar regime that agree well with the theoretical expectations of the performed numerical analysis. Finally, numerical studies for a plane mixing layer problem confirm that good accuracy is achieved for simulating a high Reynolds number flow on coarse grids.

The outline of the paper is as follows: In Section 2, we introduce the model problem and its continuous variational formulation for time-dependent NSE. In Section 3, we describe the proposed LPS approximation of the incompressible evolution NSE, commonly referred as high-order term-by-term stabilization, and we state its main properties. Section 4 is devoted to the numerical analysis (stability and error estimates) of the arising fully discrete scheme, and to the study of the asymptotic energy balance of the system. In Section 5 we present numerical studies, to test on the one hand the theoretical predictions of the performed numerical analysis and to show on the other hand the potential of the proposed 
method for simulating turbulent flows on relatively coarse grids. Section 6 states the main conclusions of the paper.

\section{Time-dependent Navier-Stokes equations: Model problem and variational formulation}

We introduce an Initial-Boundary Value Problem (IBVP) for the incompressible evolution NSE. For the sake of simplicity, we just impose homogeneous Dirichlet boundary condition on the whole boundary. More general inflow boundary conditions may be taken into account by standard lifting techniques for NSE. Also, the treatment of general non-linear wall law boundary conditions may be found in [21].

Let $[0, T]$ be the time interval, and $\Omega$ a bounded polyhedral domain in $\mathbb{R}^{d}, d=2$ or 3 , with a Lipschitz-continuous boundary $\Gamma=\partial \Omega$. The transient NSE for an incompressible fluid are given by:

$$
\begin{aligned}
& \text { Find } \boldsymbol{u}: \Omega \times(0, T) \longrightarrow \mathbb{R}^{d} \text { and } p: \Omega \times(0, T) \\
& \qquad \begin{array}{rlrl}
\partial_{t} \boldsymbol{u}+\nabla \cdot(\boldsymbol{u} \otimes \boldsymbol{u})-2 \nu \nabla \cdot D(\boldsymbol{u})+\nabla p & =\boldsymbol{R} \text { such that: } \\
\nabla \cdot \boldsymbol{u} & =0 & & \text { in } \Omega \times(0, T), \\
\boldsymbol{u} & =\mathbf{0} & & \text { in } \Omega \times(0, T), \\
\boldsymbol{u}(\boldsymbol{x}, 0) & =\boldsymbol{u}_{0}(\boldsymbol{x}) & & \text { in } \Omega,
\end{array}
\end{aligned}
$$

where $\boldsymbol{u} \otimes \boldsymbol{u}$ is the tensor function of components $u_{i} u_{j}$, and $D(\boldsymbol{u})$ is the symmetric deformation tensor given by $D(\boldsymbol{u})=(1 / 2)\left(\nabla \boldsymbol{u}+(\nabla \boldsymbol{u})^{t}\right)$. The unknowns are the velocity $\boldsymbol{u}$ and the pressure $p$ of the incompressible fluid. The data are the source term $\boldsymbol{f}$, which represents a body force per mass unit (typically the gravity), the kinematic viscosity $\nu$ of the fluid, which is a positive constant, and the initial velocity $\boldsymbol{u}_{0}$.

To define the weak formulation of problem (2.1), we need to introduce some useful notations for spaces. We consider the Sobolev spaces $H^{s}(\Omega), s \in \mathbb{R}, L^{p}(\Omega)$ and $W^{m, p}(\Omega)$, $m \in \mathbb{N}, 1 \leq p \leq \infty$. We shall use the following notation for vector-valued Sobolev spaces: $\mathbf{H}^{s}, \mathbf{L}^{p}$ and $\mathbf{W}^{m, p}$ respectively shall denote $\left[H^{s}(\Omega)\right]^{d},\left[L^{p}(\Omega)\right]^{d}$ and $\left[W^{m, p}(\Omega)\right]^{d}$ (similarly for tensor spaces of dimension $d \times d)$. Also, the parabolic Bochner function spaces $L^{p}(0, T ; X)$ and $L^{p}(0, T ; \mathbf{X})$, where $X(\mathbf{X})$ stands for a scalar (vector-valued) Sobolev space shall be denoted by $L^{p}(X)$ and $L^{p}(\mathbf{X})$, respectively. In order to give a variational formulation of problem (2.1), let us consider the velocity space:

$$
\mathbf{H}_{0}^{1}=\left[H_{0}^{1}(\Omega)\right]^{d}=\left\{\boldsymbol{w} \in\left[H^{1}(\Omega)\right]^{d}: \boldsymbol{w}=\mathbf{0} \text { on } \Gamma\right\} .
$$

This is a closed linear subspace of $\mathbf{H}^{1}$, and thus a Hilbert space endowed with the $\mathbf{H}^{1}$ norm. Thanks to Korn's inequality ( $c f$. [35]), the $\mathbf{H}^{1}$-norm is equivalent on $\mathbf{H}_{0}^{1}$ to the norm $\|\boldsymbol{w}\|_{\mathbf{H}_{0}^{1}}=\|D(\boldsymbol{w})\|_{\mathbf{L}^{2}}$. Also, let us introduce the space of divergence-free functions:

$$
\mathbf{H}_{0, \text { div }}^{1}=\left\{\boldsymbol{w} \in \mathbf{H}_{0}^{1}: \nabla \cdot \boldsymbol{w}=0 \text { a.e. in } \Omega\right\} .
$$

The space $\mathbf{H}_{0 \text {,div }}^{1}$ is a closed linear subspace of $\mathbf{H}_{0}^{1}$, and thus a Hilbert space endowed with the $\mathbf{H}^{1}$-norm. We shall consider the following variational formulation of (2.1): 
Given $\boldsymbol{f} \in L^{2}\left(\mathbf{H}^{-1}\right)$ and $\boldsymbol{u}_{0} \in \mathbf{H}^{-1}$, find $\boldsymbol{u} \in L^{\infty}\left(\mathbf{L}^{2}\right) \cap L^{2}\left(\mathbf{H}_{0, \mathrm{div}}^{1}\right), P \in L^{2}\left(L_{0}^{2}\right)$ such that:

$$
\left\{\begin{array}{l}
-\int_{0}^{T}(\boldsymbol{u}(t), \boldsymbol{v})_{\Omega} \varphi^{\prime}(t) d t-\left\langle\boldsymbol{u}_{0}, \boldsymbol{v}\right\rangle \varphi(0) \\
+\int_{0}^{T}[b(\boldsymbol{u}(t) ; \boldsymbol{u}(t), \boldsymbol{v})+a(\boldsymbol{u}(t), \boldsymbol{v})] \varphi(t) d t \\
+\int_{0}^{T}(P(t), \nabla \cdot \boldsymbol{v})_{\Omega} \varphi^{\prime}(t) d t=\int_{0}^{T}\langle\boldsymbol{f}(t), \boldsymbol{v}\rangle \varphi(t) d t
\end{array}\right.
$$

for any $\boldsymbol{v} \in \mathbf{H}_{0}^{1}, \varphi \in \mathcal{D}([0, T])$ such that $\varphi(T)=0$, where $\langle\cdot, \cdot\rangle$ stands for the duality pairing between $\mathbf{H}_{0}^{1}$ and its dual $\mathbf{H}^{-1}$. The forms $b$ and $a$ are given by:

$$
\begin{aligned}
b(\boldsymbol{w} ; \boldsymbol{u}, \boldsymbol{v}) & =\frac{1}{2}\left[(\boldsymbol{w} \cdot \nabla \boldsymbol{u}, \boldsymbol{v})_{\Omega}-(\boldsymbol{w} \cdot \nabla \boldsymbol{v}, \boldsymbol{u})_{\Omega}\right] \\
a(\boldsymbol{u}, \boldsymbol{v}) & =2 \nu(D(\boldsymbol{u}), D(\boldsymbol{v}))_{\Omega}
\end{aligned}
$$

for $\boldsymbol{u}, \boldsymbol{v}, \boldsymbol{w} \in \mathbf{H}_{0}^{1}$. Semicolons (;) are used for forms that are non-linear with respect to its first argument. Note that $b(\boldsymbol{w} ; \boldsymbol{v}, \boldsymbol{v})=0$ for all $\boldsymbol{w}, \boldsymbol{v} \in \mathbf{H}_{0}^{1}$. The physical pressure is the time derivative of the unknown $P: p=\partial_{t} P \in H^{-1}\left(L_{0}^{2}\right)=H_{0}^{1}\left(0, T ; L_{0}^{2}\right)^{\prime}$. The interest of considering $P$ as unknown instead of $p$ is that there are high technical difficulties to obtain uniform bounds for the discrete pressures in a Banach space of space-time functions (see [21], Remark 10.2), while we shall obtain uniform bounds in the Banach space $L^{\infty}\left(L^{2}\right)$ for the numerical approximation of $P$ (see estimate (4.6) of Theorem 4.3). We notice, however, that for practical computations one would approximate the physical pressure $p$, and $P$ is introduced just for the numerical analysis. Also, note that the initial condition takes place in $\mathbf{H}_{\text {div }}^{-1}$, since $\boldsymbol{u} \in C^{0}\left([0, T], \mathbf{H}_{\text {div }}^{-1}\right.$ ) (see [21], Sect. 10.2), with obvious notation.

\section{A local projection stabilization model}

Let $\left\{\mathcal{T}_{h}\right\}_{h>0}$ be a family of affine-equivalent, conforming (i.e., without hanging nodes) and regular triangulations of $\bar{\Omega}$, formed by triangles or quadrilaterals $(d=2)$, tetrahedra or hexahedra $(d=3)$. For any mesh cell $K \in \mathcal{T}_{h}$, its diameter will be denoted by $h_{K}$ and $h=\max _{K \in \mathcal{T}_{h}} h_{K}$.

Given an integer $l \geq 0$ and a mesh cell $K \in \mathcal{T}_{h}$, denote by $\mathbb{R}_{l}(K)$ either $\mathbb{P}_{l}(K)$ (i.e., the space of Lagrange polynomials of degree $\leq l$, defined on $K$ ), if the grids are formed by triangles $(d=2)$ or tetrahedra $(d=3)$, or $\mathbb{Q}_{l}(K)$ (i.e., the space of Lagrange polynomials of degree $\leq l$ on each variable, defined on $K)$, if the family of triangulations is formed by quadrilaterals $(d=2)$ or hexahedra $(d=3)$. We consider the following FE spaces for the velocity:

$$
\left\{\begin{array}{l}
Y_{h}^{l}=V_{h}^{l}(\Omega)=\left\{v_{h} \in C^{0}(\bar{\Omega}):\left.v_{h}\right|_{K} \in \mathbb{R}_{l}(K), \forall K \in \mathcal{T}_{h}\right\} \\
\mathbf{Y}_{h}^{l}=\left[Y_{h}^{l}\right]^{d}=\left\{\boldsymbol{v}_{h} \in\left[C^{0}(\bar{\Omega})\right]^{d}:\left.\boldsymbol{v}_{h}\right|_{K} \in\left[\mathbb{R}_{l}(K)\right]^{d}, \forall K \in \mathcal{T}_{h}\right\}, \\
\mathbf{X}_{h}=\mathbf{Y}_{h}^{l} \cap \mathbf{H}_{0}^{1} .
\end{array}\right.
$$

Hereafter, $\mathbf{Y}_{h}^{l}$ (resp., $Y_{h}^{l}$ ) will constitute the discrete foreground vector-valued (resp., scalar) spaces in which we will work on. 
We approximate the weak formulation (2.2) of the initial-boundary value problem (2.1) for the incompressible evolution NSE by a high-order term-by-term stabilization procedure in space $(c f$. [16]). To state this unsteady LPS discretization, consider a positive integer number $N$ and define $\Delta t=T / N, t_{n}=n \Delta t, n=0,1, \ldots, N$. We compute the approximations $\boldsymbol{u}_{h}^{n}, p_{h}^{n}$ to $\boldsymbol{u}^{n}=\boldsymbol{u}\left(\cdot, t_{n}\right)$ and $p^{n}=p\left(\cdot, t_{n}\right)$ by:

- Initialization. Set:

$$
\boldsymbol{u}_{h}^{0}=\boldsymbol{u}_{0 h}
$$

- Iteration. For $n=0,1, \ldots, N-1$ :

Given $\boldsymbol{u}_{h}^{n} \in \mathbf{X}_{h}$, find $\left(\boldsymbol{u}_{h}^{n+1}, p_{h}^{n+1}\right) \in \mathbf{X}_{h} \times \mathbb{M}_{h}$ such that:

$$
\left\{\begin{aligned}
\left(\frac{\boldsymbol{u}_{h}^{n+1}-\boldsymbol{u}_{h}^{n}}{\Delta t}, \boldsymbol{v}_{h}\right)_{\Omega}+b\left(\boldsymbol{u}_{h}^{n}, \boldsymbol{u}_{h}^{n+1}, \boldsymbol{v}_{h}\right)+a\left(\boldsymbol{u}_{h}^{n+1}, \boldsymbol{v}_{h}\right) & \\
-\left(p_{h}^{n+1}, \nabla \cdot \boldsymbol{v}_{h}\right)_{\Omega}+s_{\mathrm{conv}}\left(\boldsymbol{u}_{h}^{n}, \boldsymbol{u}_{h}^{n+1}, \boldsymbol{v}_{h}\right)+s_{\mathrm{div}}\left(\boldsymbol{u}_{h}^{n+1}, \boldsymbol{v}_{h}\right) & =\left\langle\overline{\boldsymbol{f}}^{n+1}, \boldsymbol{v}_{h}\right\rangle \\
\left(\nabla \cdot \boldsymbol{u}_{h}^{n+1}, q_{h}\right)_{\Omega}+s_{\mathrm{pres}}\left(p_{h}^{n+1}, q_{h}\right) & =0
\end{aligned}\right.
$$

for any $\left(\boldsymbol{v}_{h}, q_{h}\right) \in \mathbf{X}_{h} \times \mathbb{M}_{h}$, where $\mathbb{M}_{h}=Y_{h}^{l} \cap L_{0}^{2}, \overline{\boldsymbol{f}}^{n+1}$ is the average value of $\boldsymbol{f}$ in $\left[t_{n}, t_{n+1}\right]$ :

$$
\overline{\boldsymbol{f}}^{n+1}=\frac{1}{\Delta t} \int_{t_{n}}^{t_{n+1}} \boldsymbol{f}(s) d s,
$$

and $\boldsymbol{u}_{0 h}$ is some stable approximation to $\boldsymbol{u}_{0}$ belonging to $\mathbf{X}_{h}$, e.g., the discrete Stokes projection.

The forms $s_{\text {conv }}, s_{\text {div }}$ and $s_{\text {pres }}$ in (3.2) correspond to a high-order term-by-term stabilized method ( $c f .[16])$, and are given by:

$$
\begin{aligned}
s_{\text {conv }}\left(\boldsymbol{u}_{h}^{n}, \boldsymbol{u}_{h}^{n+1}, \boldsymbol{v}_{h}\right) & =\sum_{K \in \mathcal{T}_{h}} \tau_{\nu, K}\left(\sigma_{h}^{*}\left(\boldsymbol{u}_{h}^{n} \cdot \nabla \boldsymbol{u}_{h}^{n+1}\right), \sigma_{h}^{*}\left(\boldsymbol{u}_{h}^{n} \cdot \nabla \boldsymbol{v}_{h}\right)\right)_{K}, \\
s_{\text {div }}\left(\boldsymbol{u}_{h}^{n+1}, \boldsymbol{v}_{h}\right) & =\sum_{K \in \mathcal{T}_{h}} \tau_{d, K}\left(\sigma_{h}^{*}\left(\nabla \cdot \boldsymbol{u}_{h}^{n+1}\right), \sigma_{h}^{*}\left(\nabla \cdot \boldsymbol{v}_{h}\right)\right)_{K}, \\
s_{\text {pres }}\left(p_{h}^{n+1}, q_{h}\right) & =\sum_{K \in \mathcal{T}_{h}} \tau_{p, K}\left(\sigma_{h}^{*}\left(\nabla p_{h}^{n+1}\right), \sigma_{h}^{*}\left(\nabla q_{h}\right)\right)_{K} .
\end{aligned}
$$

Here, $\tau_{\nu, K}, \tau_{d, K}$ and $\tau_{p, K}$ are stabilization coefficients for convection, divergence and pressure gradient, respectively, and $\sigma_{h}^{*}=I d-\sigma_{h}$, where $\sigma_{h}$ is some locally stable projection or interpolation operator from $\mathbf{L}^{2}$ on the foreground vector-valued space $\mathbf{Y}_{h}^{l-1}$ (also called "buffer space" in this context): There exists a constant $C>0$ such that for any $K \in \mathcal{T}_{h}$

$$
\left\|\sigma_{h}(\boldsymbol{v})\right\|_{\mathbf{L}^{2}(K)} \leq C\|\boldsymbol{v}\|_{\mathbf{L}^{2}\left(\omega_{K}\right)}, \quad \forall \boldsymbol{v} \in \mathbf{L}^{2},
$$

where $\omega_{K}$ is the union of all mesh cells whose intersection with $K$ is not empty (note that the mesh cells are compact). Actually, $\sigma_{h}$ is globally stable in $L^{2}(\Omega)$-norm, due to the regularity of the mesh. We also assume that $\sigma_{h}$ satisfies optimal error estimates: There exists a constant $C>0$ only depending on $p, \Omega, d$ and the aspect ratio of the family of triangulations such that

$$
\left\|\boldsymbol{v}-\sigma_{h}(\boldsymbol{v})\right\|_{\mathbf{W}^{m, p}} \leq C h^{s-m+d / p-d / 2}|\boldsymbol{v}|_{\mathbf{H}^{s}},
$$


for $m \in\{0,1\}$, and $\boldsymbol{v} \in \mathbf{H}^{s}, m+1 \leq s \leq l$ (see, for instance, [22]), where $|\boldsymbol{v}|_{\mathbf{H}^{s}}$ denotes the seminorm of $\boldsymbol{v}$ in $\mathbf{H}^{s}$. In practical implementations, we choose $\sigma_{h}$ as a Scott-Zhang-like linear interpolation operator in the space $\mathbf{Y}_{h}^{l-1}$ (cf. [50]). In case of (3.4), $\sigma_{h}$ denotes an operator between the scalar spaces $L^{2}$ and $Y_{h}^{l-1}$, but we use the same notation for the sake of simplicity. Actually, if needed, specific stabilizations for convection, divergence and pressure gradient may be used, through different approximation operators. For the subsequent numerical analysis, we need the following technical hypothesis on the stabilization coefficients:

Hypothesis 3.1. The stabilization coefficients $\tau_{p, K}, \tau_{d, K}$ and $\tau_{\nu, K}$ satisfy the following conditions:

$$
\alpha_{1} h_{K}^{2} \leq \tau_{p, K} \leq \alpha_{2} h_{K}^{2}, \quad 0<\tau_{d, K} \leq \beta, \quad 0<\tau_{\nu, K} \leq \gamma h_{K}^{2},
$$

for all $K \in \mathcal{T}_{h}$, and some positive constants $\alpha_{1}, \alpha_{2}, \beta, \gamma$ independent of $h$.

Remark 3.2. The proposed spatial discretization is a term-by-term interpolation-stabilized method with increased accuracy with respect to the pure penalty term-by-term stabilized method (cf. [15]). It presents the same structure of the Streamline Derivative-based (SDbased) LPS model (cf. [10, 39]), but it differs from it because at the same time it uses continuous buffer functions, it does not need enriched FE spaces, it does not need a projection with local orthogonality properties, and it does not need different nested meshes.

The high-order term-by-term stabilization procedure by using a Scott-Zhang-like interpolation operator has been successfully applied to the Oseen problem (cf. [16]). Moreover, it has been extended to the evolution NSE (cf. [17]) and the primitive equations of the ocean (cf. [20]), and recently shown by numerical experiments that, since this method is only approximately consistent, the addition of a multi-scale Smagorinsky term to the high-order term-by-term stabilization scheme can help to counter-balance the accumulation of sub-grid energy due to its diffusive nature, providing slight additional accuracy (cf. [19]). This recently proposed projection-based VMS turbulence model (called VMS-S model, see [1, 18, 19, 21, 49]) has thus a dual nature, as it results in a combination of (high-order term-by-term) stabilization and (projection) VMS-LES modeling. The analysis of the multi-scale Smagorinsky term may be found in [1, 18, 19, 21, 49].

Remark 3.3. The chosen discretization in time gives rise to a semi-implicit Euler scheme, since the discretization of the convection terms is semi-implicit, while that of the remaining terms is implicit. Note that scheme (3.2) consists of a high-order discretization method in space (optimal for smooth solutions, as we will prove in Section 4.3 by an error analysis) although, for the sake of simplicity, we shall only consider a first-order discretization in time to perform the numerical analysis. This approach allows to achieve the stability of the scheme in $L^{\infty}\left(\mathbf{L}^{2}\right) \cap L^{2}\left(\mathbf{H}^{1}\right)$ for the velocities. These stability properties are also shared by more general $\theta$-schemes (e.g., the Crank-Nicolson scheme).

\section{Analysis of the discrete model}

In this section, we perform the numerical analysis of the proposed unsteady model (3.2), which we will call in the sequel STAB model. For technical reasons, we assume throughout the work that the family of triangulations $\left\{\mathcal{T}_{h}\right\}_{h>0}$ is uniformly regular (also called quasiuniform): There exist two constants $C_{1}, C_{2}>0$ independent of $h$ such that

$$
\forall K \in \mathcal{T}_{h}, \quad C_{1} h \leq h_{K} \leq C_{2} \rho_{K},
$$


where $\rho_{K}$ is the diameter of the ball inscribed in $K$. Actually, this technical hypothesis may be relaxed to the more general case of regular grids, but we keep it to focus the analysis on the new aspects of the method, and to not unnecessarily lengthen it.

\subsection{Technical background}

This section provides some technical results that are required for the numerical analysis. Throughout the paper, we shall denote by $C, C_{1}, C_{2}, \ldots$ constants that may vary from a line to another, but which are always independent of $h, \Delta t$, and $\nu$. We define the scalar product:

$$
(\cdot, \cdot)_{\tau}: L^{2} \times L^{2} \rightarrow \mathbb{R}, \quad(f, g)_{\tau}=\sum_{K \in \mathcal{T}_{h}} \tau_{K}(f, g)_{K},
$$

where $\tau$ denotes either $\tau_{\nu}, \tau_{d}$, or $\tau_{p}$, and its associated norm:

$$
\|f\|_{\tau}=(f, f)_{\tau}^{1 / 2} .
$$

Lemma 4.1. Assume that Hypothesis 3.1 holds. Then, for all $z, g \in L^{2}$, the following conditions are satisfied:

$$
\begin{gathered}
C_{1} \sum_{K \in \mathcal{T}_{h}} h_{K}^{2}\|z\|_{L^{2}(K)}^{2} \leq\|z\|_{\tau_{p}}^{2} \leq C_{2} \sum_{K \in \mathcal{T}_{h}} h_{K}^{2}\|z\|_{L^{2}(K)}^{2}, \\
\|z\|_{\tau_{d}}^{2} \leq C_{3} \sum_{K \in \mathcal{T}_{h}}\|z\|_{L^{2}(K)}^{2}, \quad\|z\|_{\tau_{\nu}}^{2} \leq C_{4} \sum_{K \in \mathcal{T}_{h}} h_{K}^{2}\|z\|_{L^{2}(K)}^{2},
\end{gathered}
$$

and:

$$
\begin{gathered}
\left\|\sigma_{h}^{*}(g)\right\|_{\tau_{p}} \leq C_{5} h\|g\|_{L^{2}}, \\
\left\|\sigma_{h}^{*}(g)\right\|_{\tau_{d}} \leq C_{6}\|g\|_{L^{2}}, \quad\left\|\sigma_{h}^{*}(g)\right\|_{\tau_{\nu}} \leq C_{7} h\|g\|_{L^{2}} .
\end{gathered}
$$

Proof. Estimates (4.1) and (4.2) immediately follow from (3.8).

Let $g \in L^{2}$. By applying the second part of (4.1) to $\sigma_{h}^{*}(g)$, we obtain:

$$
\left\|\sigma_{h}^{*}(g)\right\|_{\tau_{p}}^{2} \leq C_{2} \sum_{K \in \mathcal{T}_{h}} h_{K}^{2}\left\|\sigma_{h}^{*}(g)\right\|_{L^{2}(K)}^{2} \leq C_{2} h^{2}\left\|\sigma_{h}^{*}(g)\right\|_{L^{2}}^{2} \leq C h^{2}\|g\|_{L^{2}}^{2},
$$

where we have used the global version of stability estimate (3.6), due to the regularity of the mesh. Similarly, by applying (4.2) to $\sigma_{h}^{*}(g)$, we obtain:

$$
\left\|\sigma_{h}^{*}(g)\right\|_{\tau_{d}}^{2} \leq C\|g\|_{L^{2}}^{2}, \quad\left\|\sigma_{h}^{*}(g)\right\|_{\tau_{\nu}}^{2} \leq C h^{2}\|g\|_{L^{2}}^{2} .
$$

Thus, the estimates (4.3) and (4.4) can be deduced.

We next state a specific discrete inf-sup condition for the stabilized approximation that is essential for the stability of method (3.2). The main difficulty in its proof stems from the fact that the interpolation operator $\sigma_{h}$ takes values in $\mathbf{Y}_{h}^{l-1}$, thus reducing the effective number of degrees of freedom (d.o.f.) of the foreground velocity space $\mathbf{Y}_{h}^{l}$.

Lemma 4.2. Assume that Hypothesis 3.1 holds. Then, we have the following inf-sup condition:

$$
\forall q_{h} \in \mathbb{M}_{h}, \quad\left\|q_{h}\right\|_{L^{2}} \leq C\left(\sup _{\boldsymbol{v}_{h} \in \mathbf{X}_{h}} \frac{\left(\nabla \cdot \boldsymbol{v}_{h}, q_{h}\right)_{\Omega}}{\left\|D\left(\boldsymbol{v}_{h}\right)\right\|_{\mathbf{L}^{2}}}+\left\|\sigma_{h}^{*}\left(\nabla q_{h}\right)\right\|_{\tau_{p}}\right),
$$

for some positive constant $C$ independent of $h$. 
The proof of this lemma can be derived from [16]. Note that the discrete inf-sup condition (4.5) can be extended to a more complex condition that holds for a regular family of triangulations.

\subsection{Existence and stability results}

Let us first show results on existence, uniqueness of a solution and the stability of method (3.2). To state them, we shall consider the following discrete functions:

- $\boldsymbol{u}_{h}$ is the piecewise linear in time function with values on $\mathbf{X}_{h}$ such that $\boldsymbol{u}_{h}\left(t_{n}\right)=\boldsymbol{u}_{h}^{n}$,

- $\widetilde{p_{h}}$ is the piecewise constant in time function that takes the value $p_{h}^{n+1}$ on $\left(t_{n}, t_{n+1}\right)$, - $P_{h}(t)=\int_{0}^{t} \widetilde{p_{h}}(s) d s$.

For simplicity of notation, we do not make explicit the dependence of these functions upon $\Delta t$.

Theorem 4.3. Assume that Hypothesis 3.1 holds, and let $\boldsymbol{f} \in L^{2}\left(\mathbf{H}^{-1}\right), \boldsymbol{u}_{0} \in \mathbf{L}^{2}$. Then, problem (3.2) admits a unique solution that satisfies the estimate:

$$
\left\|\boldsymbol{u}_{h}\right\|_{L^{\infty}\left(\mathbf{L}^{2}\right)}+\sqrt{\nu}\left\|D\left(\boldsymbol{u}_{h}\right)\right\|_{L^{2}\left(\mathbf{L}^{2}\right)}+\left\|P_{h}\right\|_{L^{\infty}\left(L^{2}\right)} \leq C\left(\left\|\boldsymbol{u}_{0}\right\|_{\mathbf{L}^{2}}+\frac{1}{\sqrt{\nu}}\|\boldsymbol{f}\|_{L^{2}\left(\mathbf{H}^{-1}\right)}\right),
$$

where $C>0$ is a constant independent of $h$ and $\Delta t$.

The proof of this theorem can be directly derived by the one performed for the VMS-S model in [18].

Remark 4.4. In [18], the following stability estimate was derived:

$$
\left\|\sigma_{h}^{*}\left(\boldsymbol{u}_{h} \cdot \nabla \boldsymbol{u}_{h}\right)\right\|_{L^{2}\left(\tau_{\nu}\right)} \leq C\left(\left\|\boldsymbol{u}_{0}\right\|_{\mathbf{L}^{2}}+\frac{1}{\sqrt{\nu}}\|\boldsymbol{f}\|_{L^{2}\left(\mathbf{H}^{-1}\right)}\right) .
$$

Thus, the convective stabilization term provides some control as $\nu \rightarrow 0$ of a range of small scales of the convective derivative, actually those scales that are not representable in the buffer space $\mathbf{Y}_{h}^{l-1}$ by means of the operator $\sigma_{h}$. The large scales $\sigma_{h}\left(\boldsymbol{u}_{h}(t) \cdot \nabla \boldsymbol{u}_{h}(t)\right)$ of the convective derivative, which can be represented in the buffer space, are directly bounded in a weak sense by the convection term in discretization (3.2) for a large class of operators $\sigma_{h}$ (see Remark 3.4 in [17]).

\subsection{Error estimates}

We next prove error estimates for the approximation of the unsteady NSE (2.2) by the discrete model (3.2). We obtain these estimates for rather general fluid viscosities (and not just for relatively high viscosities, as in the steady case, see [19, 49]). If the flow is regular enough, we obtain convergence of optimal order, and the order decreases with the regularity. As already mentioned in the introduction, to the best of our knowledge, in the literature there is no proof concerning a-priori error estimates for the fully discrete incompressible evolutionary NSE with local projection as a stabilization in space.

To state this result, we start with the discrete version of the Gronwall's lemma: 
Lemma 4.5 ([21], Lemma 10.4). Let $\left\{\alpha_{n}\right\}_{n=0}^{N},\left\{\beta_{n}\right\}_{n=0}^{N}$ be two finite sequences of nonnegative real numbers such that:

$$
\left(1-C_{n} \Delta t\right) \alpha_{n+1} \leq\left(1+D_{n} \Delta t\right) \alpha_{n}+\beta_{n}, \text { for } n=0,1, \ldots, N-1,
$$

for two finite sequences of non-negative real numbers $\left\{C_{n}\right\}_{n=0}^{N},\left\{D_{n}\right\}_{n=0}^{N}$. Assume $\Delta t \leq 1 /\left(2 \max _{n=0,1, \ldots, N-1} C_{n}\right)$. Then:

$$
\max _{n=0,1, \ldots, N} \alpha_{n} \leq \alpha_{0} e^{2 \Delta t S_{N}}+2 e^{2 \Delta t S_{N-1}} \sum_{n=0}^{N-1} \beta_{n},
$$

where $S_{N}=\sum_{n=0}^{N-1}\left(C_{n}+D_{n}\right)$.

We are now in position to prove the following error estimate result:

Theorem 4.6. Assume that Hypothesis 3.1 holds, the data verify $\boldsymbol{f} \in C^{0}\left(\mathbf{H}^{-1}\right), \partial_{t} \boldsymbol{f} \in$ $L^{2}\left(\mathbf{H}^{-1}\right), \boldsymbol{u}_{0} \in \mathbf{H}^{s+1}$, and that the solution $(\boldsymbol{u}, p)$ of the unsteady NSE (2.2) has augmented regularity, i.e., $(\boldsymbol{u}, p) \in C^{0}\left(\mathbf{H}^{s+1}\right) \times C^{0}\left(H^{s}\right), 2 \leq s \leq l$, such that $\partial_{t t} \boldsymbol{u} \in L^{2}\left(\mathbf{L}^{2}\right)$. Assume in addition that there is a constant $C$ independent of $h$ and $\Delta t$ such that $C h \leq \Delta t$ and that $\left\|\boldsymbol{u}_{0}-\boldsymbol{u}_{0 h}\right\|_{\mathbf{H}^{1}}=\mathcal{O}\left(h^{s}\right)$. Then, the following error estimate for a solution $\left\{\boldsymbol{u}_{h}, p_{h}\right\}$ of the fully discrete STAB model (3.2) holds:

$$
\begin{aligned}
& \left\|\boldsymbol{u}-\boldsymbol{u}_{h}\right\|_{\ell^{\infty}\left(\mathbf{L}^{2}\right)}+\sqrt{\nu}\left\|D\left(\boldsymbol{u}-\boldsymbol{u}_{h}\right)\right\|_{\ell^{2}\left(\mathbf{L}^{2}\right)}+\left\|\widetilde{P}-P_{h}\right\|_{\ell^{\infty}\left(L^{2}\right)} \\
& \quad \leq \sqrt{\widetilde{C} e^{\widetilde{C}}}\left[h^{s}\left(\|\boldsymbol{u}\|_{L^{\infty}\left(\mathbf{H}^{s+1}\right)}+\|p\|_{L^{\infty}\left(H^{s}\right)}+\|\boldsymbol{u}\|_{L^{\infty}\left(\mathbf{H}^{s+1}\right)}^{2}+1\right)+\Delta t\right],
\end{aligned}
$$

for $\widetilde{C}=C(T)\left(1+\nu^{-1}\right)\|\boldsymbol{u}\|_{L^{\infty}\left(\mathbf{H}^{s+1}\right)}^{2}$ and $C(T)>0$ an increasing function of $T$ independent of $h$ and $\Delta t$, where we are using the following notation:

$$
\begin{aligned}
\left\|\boldsymbol{u}-\boldsymbol{u}_{h}\right\|_{\ell^{\infty}\left(\mathbf{L}^{2}\right)} & =\max _{n=1, \ldots, N}\left\|\boldsymbol{u}^{n}-\boldsymbol{u}_{h}^{n}\right\|_{\mathbf{L}^{2}}, \\
\left\|D\left(\boldsymbol{u}-\boldsymbol{u}_{h}\right)\right\|_{\ell^{2}\left(\mathbf{L}^{2}\right)} & =\left[\sum_{n=1}^{N} \Delta t\left\|D\left(\boldsymbol{u}^{n}-\boldsymbol{u}_{h}^{n}\right)\right\|_{\mathbf{L}^{2}}^{2}\right]^{1 / 2}, \\
\left\|\widetilde{P}-P_{h}\right\|_{\ell^{\infty}\left(L^{2}\right)} & =\max _{n=1, \ldots, N}\left\|\widetilde{P}^{n}-P_{h}^{n}\right\|_{L^{2}},
\end{aligned}
$$

being $\widetilde{P}=\int_{0}^{t} \widetilde{p}(\cdot, s) d s$, with $\widetilde{p}$ the piecewise constant in time function that takes the value $p^{n+1}$ on $\left(t_{n}, t_{n+1}\right)$, and $\widetilde{P}^{n}=\widetilde{P}\left(\cdot, t_{n}\right), P_{h}^{n}=P_{h}\left(t_{n}\right)$.

Proof. The proof will be split into four main steps. Throughout the proof, it will be assumed that $0<h, \Delta t \leq 1$.

Step 1: Error equation. We consider an approximation $\widehat{\boldsymbol{u}}_{h}^{n}=R_{h} \boldsymbol{u}^{n} \in \mathbf{X}_{h} \subset \mathbf{Y}_{h}^{l}$ of $\boldsymbol{u}^{n}=\boldsymbol{u}\left(\cdot, t_{n}\right) \in \mathbf{H}_{0}^{1}$ satisfying:

$$
\left(\boldsymbol{u}^{n}-\widehat{\boldsymbol{u}}_{h}^{n}, \boldsymbol{v}_{h}\right)_{\Omega}=0, \quad \forall \boldsymbol{v}_{h} \in \mathbf{Y}_{h}^{l-1}, n=0,1, \ldots N .
$$

Note that such interpolant $R_{h}$ exists, and satisfies optimal approximation properties as the standard nodal Lagrange interpolant ( $c f$. [22]): There exists a constant $C>0$ only depending on $p, \Omega, d$ and the aspect ratio of the family of triangulations such that

$$
\left\|\boldsymbol{u}^{n}-\widehat{\boldsymbol{u}}_{h}^{n}\right\|_{\mathbf{W}^{m, p}} \leq C h^{s+1-m+d / p-d / 2}\left|\boldsymbol{u}^{n}\right|_{\mathbf{H}^{s+1}}, \quad n=0,1, \ldots N,
$$


for $m \in\{0,1\}$. This has been proved in [16], Lemma 3.7 (note that, due to the required augmented regularity, the solution $(\boldsymbol{u}, p)$ is continuous in space, by Sobolev imbedding theorem). Also, let $\widehat{p}_{h}^{n}=T_{h} p^{n} \in \mathbb{M}_{h}\left(p^{n}=p\left(\cdot, t_{n}\right)\right)$ with $T_{h}$ the standard FE interpolation operator, that satisfies optimal approximation properties ( $c f$. [22]):

There exists a constant $C>0$ only depending on $p, \Omega, d$ and the aspect ratio of the family of triangulations such that

$$
\left\|p^{n}-\widehat{p}_{h}^{n}\right\|_{\mathbf{W}^{m, p}} \leq C h^{s-m+d / p-d / 2}\left|p^{n}\right|_{\mathbf{H}^{s}}, \quad n=0,1, \ldots N,
$$

for $m \in\{0,1\}$.

Let us define the errors in velocity and pressure by $\boldsymbol{e}_{h}^{n}=\widehat{\boldsymbol{u}}_{h}^{n}-\boldsymbol{u}_{h}^{n}, \lambda_{h}^{n}=\widehat{p}_{h}^{n}-p_{h}^{n}$, respectively. As $\partial_{t t} \boldsymbol{u} \in L^{2}\left(\mathbf{L}^{2}\right)$, then $\partial_{t} \boldsymbol{u} \in C^{0}\left([0, T], \mathbf{L}^{2}\right)$. Also, as $\boldsymbol{f} \in C^{0}\left(\mathbf{H}^{-1}\right),(\boldsymbol{u}, p) \in C^{0}\left(\mathbf{H}^{s+1}\right) \times$ $C^{0}\left(H^{s}\right)$ with $s \geq 2$, then the unsteady NSE (2.2) yields:

$$
\left\{\begin{aligned}
\left(\partial_{t} \boldsymbol{u}(t), \boldsymbol{v}\right)_{\Omega}+b(\boldsymbol{u}(t) ; \boldsymbol{u}(t), \boldsymbol{v})+a(\boldsymbol{u}(t), \boldsymbol{v})-(p(t), \nabla \cdot \boldsymbol{v})_{\Omega} & =\langle\boldsymbol{f}(t), \boldsymbol{v}\rangle, \\
(\nabla \cdot \boldsymbol{u}(t), q)_{\Omega} & =0 \\
\boldsymbol{u}(0) & =\boldsymbol{u}_{0}
\end{aligned}\right.
$$

for any $(\boldsymbol{v}, q) \in \mathbf{H}_{0}^{1} \times L_{0}^{2}$, for all $t \in[0, T]$. Subtracting (4.12) at $t=t_{n+1}$ from (3.2), we obtain the error equation:

$$
\begin{aligned}
&\left(\frac{\boldsymbol{e}_{h}^{n+1}-\boldsymbol{e}_{h}^{n}}{\Delta t}, \boldsymbol{v}_{h}\right)_{\Omega}+b\left(\widehat{\boldsymbol{u}}_{h}^{n}, \widehat{\boldsymbol{u}}_{h}^{n+1}, \boldsymbol{v}_{h}\right)-b\left(\boldsymbol{u}_{h}^{n}, \boldsymbol{u}_{h}^{n+1}, \boldsymbol{v}_{h}\right)+a\left(\boldsymbol{e}_{h}^{n+1}, \boldsymbol{v}_{h}\right)-\left(\lambda_{h}^{n+1}, \nabla \cdot \boldsymbol{v}_{h}\right)_{\Omega} \\
&+\left(\nabla \cdot \boldsymbol{e}_{h}^{n+1}, q_{h}\right)_{\Omega}=\left\langle\varepsilon_{v h}^{n+1}, \boldsymbol{v}_{h}\right\rangle+\left\langle\varepsilon_{q h}^{n+1}, q_{h}\right\rangle+s_{\mathrm{conv}}\left(\boldsymbol{u}_{h}^{n}, \boldsymbol{u}_{h}^{n+1}, \boldsymbol{v}_{h}\right) \\
&+s_{\operatorname{div}}\left(\boldsymbol{u}_{h}^{n+1}, \boldsymbol{v}_{h}\right)+s_{\operatorname{pres}}\left(p_{h}^{n+1}, q_{h}\right),
\end{aligned}
$$

for all $\left(\boldsymbol{v}_{h}, q_{h}\right) \in \mathbf{X}_{h} \times \mathbb{M}_{h}$, where $\varepsilon_{v h}^{n+1} \in \mathbf{H}^{-1}$ and $\varepsilon_{q h}^{n+1} \in L^{2}$ define the consistency error:

$$
\begin{aligned}
\left\langle\varepsilon_{v h}^{n+1}, \boldsymbol{v}_{h}\right\rangle= & -\left(\partial_{t} \boldsymbol{u}^{n+1}-\frac{\widehat{\boldsymbol{u}}_{h}^{n+1}-\widehat{\boldsymbol{u}}_{h}^{n}}{\Delta t}, \boldsymbol{v}_{h}\right)_{\Omega}-b\left(\boldsymbol{u}^{n+1} ; \boldsymbol{u}^{n+1}, \boldsymbol{v}_{h}\right)+b\left(\widehat{\boldsymbol{u}}_{h}^{n}, \widehat{\boldsymbol{u}}_{h}^{n+1}, \boldsymbol{v}_{h}\right) \\
& +a\left(\widehat{\boldsymbol{e}}_{h}^{n+1}, \boldsymbol{v}_{h}\right)-\left(\widehat{\lambda}_{h}^{n+1}, \nabla \cdot \boldsymbol{v}_{h}\right)_{\Omega}-\left\langle\overline{\boldsymbol{f}}^{n+1}-\boldsymbol{f}^{n+1}, \boldsymbol{v}_{h}\right\rangle, \\
\left\langle\varepsilon_{q h}^{n+1}, q_{h}\right\rangle= & \left(\nabla \cdot \widehat{\boldsymbol{e}}_{h}^{n+1}, q_{h}\right)_{\Omega},
\end{aligned}
$$

and we have defined $\widehat{\boldsymbol{e}}_{h}^{n+1}=\widehat{\boldsymbol{u}}_{h}^{n+1}-\boldsymbol{u}^{n+1}, \widehat{\lambda}_{h}^{n+1}=\widehat{p}_{h}^{n+1}-p^{n+1}, \boldsymbol{f}^{n+1}=\boldsymbol{f}\left(\cdot, t_{n+1}\right)$.

Step 2: Velocity estimate. Setting $\boldsymbol{v}_{h}=\boldsymbol{e}_{h}^{n+1}, q_{h}=\lambda_{h}^{n+1}$ in (4.13), using:

$$
\begin{aligned}
2\left(\boldsymbol{e}_{h}^{n+1}-\boldsymbol{e}_{h}^{n}, \boldsymbol{e}_{h}^{n+1}\right)_{\Omega} & =\left\|\boldsymbol{e}_{h}^{n+1}\right\|_{\mathbf{L}^{2}}^{2}-\left\|\boldsymbol{e}_{h}^{n}\right\|_{\mathbf{L}^{2}}^{2}+\left\|\boldsymbol{e}_{h}^{n+1}-\boldsymbol{e}_{h}^{n}\right\|_{\mathbf{L}^{2}}^{2}, \\
b\left(\boldsymbol{e}_{h}^{n}, \widehat{\boldsymbol{u}}_{h}^{n+1}, \boldsymbol{e}_{h}^{n+1}\right) & =b\left(\widehat{\boldsymbol{u}}_{h}^{n}, \widehat{\boldsymbol{u}}_{h}^{n+1}, \boldsymbol{e}_{h}^{n+1}\right)-b\left(\boldsymbol{u}_{h}^{n}, \boldsymbol{u}_{h}^{n+1}, \boldsymbol{e}_{h}^{n+1}\right),
\end{aligned}
$$

applying Young's inequality, Hölder's inequality, Korn's inequality, and the Sobolev imbed- 
ding theorem yields:

$$
\begin{aligned}
&\left\|\boldsymbol{e}_{h}^{n+1}\right\|_{\mathbf{L}^{2}}^{2}-\left\|\boldsymbol{e}_{h}^{n}\right\|_{\mathbf{L}^{2}}^{2}+\left\|\boldsymbol{e}_{h}^{n+1}-\boldsymbol{e}_{h}^{n}\right\|_{\mathbf{L}^{2}}^{2}+4 \nu \Delta t\left\|D\left(\boldsymbol{e}_{h}^{n+1}\right)\right\|_{\mathbf{L}^{2}}^{2} \\
&=\quad 2 \Delta t\left[\left\langle\varepsilon_{v h}^{n+1}, \boldsymbol{e}_{h}^{n+1}\right\rangle+\left\langle\varepsilon_{q h}^{n+1}, \lambda_{h}^{n+1}\right\rangle-b\left(\boldsymbol{e}_{h}^{n}, \widehat{\boldsymbol{u}}_{h}^{n+1}, \boldsymbol{e}_{h}^{n+1}\right)\right] \\
&+2 \Delta t\left[s_{\mathrm{conv}}\left(\boldsymbol{u}_{h}^{n}, \boldsymbol{u}_{h}^{n+1}, \boldsymbol{e}_{h}^{n+1}\right)+s_{\operatorname{div}}\left(\boldsymbol{u}_{h}^{n+1}, \boldsymbol{e}_{h}^{n+1}\right)+s_{\text {pres }}\left(p_{h}^{n+1}, \lambda_{h}^{n+1}\right)\right] \\
& \leq \Delta t\left(\nu^{-1}\left\|\varepsilon_{v h}^{n+1}\right\|_{\mathbf{H}^{-1}}^{2}+\nu\left\|D\left(\boldsymbol{e}_{h}^{n+1}\right)\right\|_{\mathbf{L}^{2}}^{2}\right) \\
&+C \Delta t\left\|\boldsymbol{e}_{h}^{n}\right\|_{\mathbf{L}^{2}}\left(\left\|D\left(\widehat{\boldsymbol{u}}_{h}^{n+1}\right)\right\|_{\mathbf{L}^{3}}+\left\|\widehat{\boldsymbol{u}}_{h}^{n+1}\right\|_{\mathbf{L}^{\infty}}\right)\left\|D\left(\boldsymbol{e}_{h}^{n+1}\right)\right\|_{\mathbf{L}^{2}} \\
&+2 \Delta t\left[\left\langle\varepsilon_{q h}^{n+1}, \lambda_{h}^{n+1}\right\rangle+s_{\operatorname{conv}}\left(\boldsymbol{u}_{h}^{n}, \boldsymbol{u}_{h}^{n+1}, \boldsymbol{e}_{h}^{n+1}\right)+s_{\operatorname{div}}\left(\boldsymbol{u}_{h}^{n+1}, \boldsymbol{e}_{h}^{n+1}\right)+s_{\mathrm{pres}}\left(p_{h}^{n+1}, \lambda_{h}^{n+1}\right)\right] \\
& \leq \Delta t\left(\nu^{-1}\left\|\varepsilon_{v h}^{n+1}\right\|_{\mathbf{H}^{-1}}^{2}+2 \nu\left\|D\left(\boldsymbol{e}_{h}^{n+1}\right)\right\|_{\mathbf{L}^{2}}^{2}+C \nu^{-1}\|\boldsymbol{u}\|_{L^{\infty}\left(\mathbf{H}^{s+1}\right)}^{2}\left\|\boldsymbol{e}_{h}^{n}\right\|_{\mathbf{L}^{2}}^{2}\right) \\
&+2 \Delta t\left[\left\langle\varepsilon_{q h}^{n+1}, \lambda_{h}^{n+1}\right\rangle+s_{\operatorname{conv}}\left(\boldsymbol{u}_{h}^{n}, \boldsymbol{u}_{h}^{n+1}, \boldsymbol{e}_{h}^{n+1}\right)+s_{\operatorname{div}}\left(\boldsymbol{u}_{h}^{n+1}, \boldsymbol{e}_{h}^{n+1}\right)+s_{\mathrm{pres}}\left(p_{h}^{n+1}, \lambda_{h}^{n+1}\right)\right],
\end{aligned}
$$

where in the last step we have used:

$$
\begin{aligned}
\left\|D\left(\widehat{\boldsymbol{u}}_{h}^{n}\right)\right\|_{\mathbf{L}^{3}} & \leq\left\|D\left(\boldsymbol{u}^{n}\right)\right\|_{\mathbf{L}^{3}}+\left\|D\left(\boldsymbol{u}^{n}\right)-D\left(\widehat{\boldsymbol{u}}_{h}^{n}\right)\right\|_{\mathbf{L}^{3}} \leq C\left\|\boldsymbol{u}^{n}\right\|_{\mathbf{H}^{s+1}}+C h^{s-d / 6}\left\|\boldsymbol{u}^{n}\right\|_{\mathbf{H}^{s+1}} \\
& \leq C\|\boldsymbol{u}\|_{L^{\infty}\left(\mathbf{H}^{s+1}\right)}, \\
\left\|\widehat{\boldsymbol{u}}_{h}^{n}\right\|_{\mathbf{L}^{\infty}} & \leq\left\|\boldsymbol{u}^{n}\right\|_{\mathbf{L}^{\infty}}+\left\|\boldsymbol{u}^{n}-\widehat{\boldsymbol{u}}_{h}^{n}\right\|_{\mathbf{L}^{\infty}} \leq C\left\|\boldsymbol{u}^{n}\right\|_{\mathbf{H}^{s+1}}+C h^{s+1-d / 2}\left\|\boldsymbol{u}^{n}\right\|_{\mathbf{H}^{s+1}} \\
4.14) & \leq C\|\boldsymbol{u}\|_{L^{\infty}\left(\mathbf{H}^{s+1}\right)},
\end{aligned}
$$

for all $n=0,1, \ldots, N$, which follows by (4.10), and Sobolev imbedding theorem, since $\boldsymbol{u} \in C^{0}\left(\mathbf{H}^{s+1}\right)$ with $s \geq 2$, and thus, in particular, $\boldsymbol{u} \in C^{0}\left(\mathbf{C}^{1}(\bar{\Omega})\right)$. It follows that:

$$
\begin{aligned}
& \left\|\boldsymbol{e}_{h}^{n+1}\right\|_{\mathbf{L}^{2}}^{2}+\left\|\boldsymbol{e}_{h}^{n+1}-\boldsymbol{e}_{h}^{n}\right\|_{\mathbf{L}^{2}}^{2}+2 \nu \Delta t\left\|D\left(\boldsymbol{e}_{h}^{n+1}\right)\right\|_{\mathbf{L}^{2}}^{2} \\
& \leq \quad\left(1+C \nu^{-1} \Delta t\|\boldsymbol{u}\|_{L^{\infty}\left(\mathbf{H}^{s+1}\right)}^{2}\right)\left\|\boldsymbol{e}_{h}^{n}\right\|_{\mathbf{L}^{2}}^{2}+\nu^{-1} \Delta t\left\|\varepsilon_{v h}^{n+1}\right\|_{\mathbf{H}^{-1}}^{2}+2 \Delta t\left\langle\varepsilon_{q h}^{n+1}, \lambda_{h}^{n+1}\right\rangle \\
& \quad+2 \Delta t\left[s_{\text {conv }}\left(\boldsymbol{u}_{h}^{n}, \boldsymbol{u}_{h}^{n+1}, \boldsymbol{e}_{h}^{n+1}\right)+s_{\text {div }}\left(\boldsymbol{u}_{h}^{n+1}, \boldsymbol{e}_{h}^{n+1}\right)+s_{\text {pres }}\left(p_{h}^{n+1}, \lambda_{h}^{n+1}\right)\right] .
\end{aligned}
$$

Note that, by divergence theorem and (4.9), one has:

$$
\left\langle\varepsilon_{q h}^{n+1}, \lambda_{h}^{n+1}\right\rangle=\left(\nabla \cdot \widehat{\boldsymbol{e}}_{h}^{n+1}, \lambda_{h}^{n+1}\right)_{\Omega}=-\left(\widehat{\boldsymbol{e}}_{h}^{n+1}, \sigma_{h}^{*}\left(\nabla \lambda_{h}^{n+1}\right)\right)_{\Omega} .
$$

By using the Cauchy-Schwarz inequality, Hypothesis 3.1, the local version of error estimate (4.10), the regularity of the grid, and Young's inequality in the last line, we obtain:

$$
\begin{aligned}
\left\langle\varepsilon_{q h}^{n+1}, \lambda_{h}^{n+1}\right\rangle & =-\left(\widehat{\boldsymbol{e}}_{h}^{n+1}, \sigma_{h}^{*}\left(\nabla \lambda_{h}^{n+1}\right)\right)_{\Omega} \leq \sum_{K \in \mathcal{T}_{h}}\left\|\widehat{\boldsymbol{e}}_{h}^{n+1}\right\|_{\mathbf{L}^{2}(K)}\left\|\sigma_{h}^{*}\left(\nabla \lambda_{h}^{n+1}\right)\right\|_{\mathbf{L}^{2}(K)} \\
& \leq\left\|\sigma_{h}^{*}\left(\nabla \lambda_{h}^{n+1}\right)\right\|_{\tau_{p}}\left(\sum_{K \in \mathcal{T}_{h}} \frac{1}{\tau_{p, K}}\left\|\widehat{\boldsymbol{e}}_{h}^{n+1}\right\|_{\mathbf{L}^{2}(K)}^{2}\right)^{1 / 2} \\
& \leq \frac{1}{\sqrt{\alpha_{1}}}\left\|\sigma_{h}^{*}\left(\nabla \lambda_{h}^{n+1}\right)\right\|_{\tau_{p}}\left(\sum_{K \in \mathcal{T}_{h}} \frac{1}{h_{K}^{2}}\left\|\widehat{\boldsymbol{e}}_{h}^{n+1}\right\|_{\mathbf{L}^{2}(K)}\right)^{1 / 2} \\
& \leq \frac{C}{\sqrt{\alpha_{1}}}\left\|\sigma_{h}^{*}\left(\nabla \lambda_{h}^{n+1}\right)\right\|_{\tau_{p}}\|\boldsymbol{u}\|_{L^{\infty}\left(\mathbf{H}^{s+1}\right)} h^{s} \\
& \leq \frac{1}{4}\left\|\sigma_{h}^{*}\left(\nabla \lambda_{h}^{n+1}\right)\right\|_{\tau_{p}}^{2}+C\|\boldsymbol{u}\|_{L^{\infty}\left(\mathbf{H}^{s+1}\right)}^{2} h^{2 s}
\end{aligned}
$$


As $p_{h}^{n+1}=\widehat{p}_{h}^{n+1}-\lambda_{h}^{n+1}$, we have:

$$
s_{\text {pres }}\left(p_{h}^{n+1}, \lambda_{h}^{n+1}\right)=s_{\text {pres }}\left(\widehat{p}_{h}^{n+1}, \lambda_{h}^{n+1}\right)-\left\|\sigma_{h}^{*}\left(\nabla \lambda_{h}^{n+1}\right)\right\|_{\tau_{p}}^{2} .
$$

Using Young's inequality to estimate $s_{\text {pres }}\left(\widehat{p}_{h}^{n+1}, \lambda_{h}^{n+1}\right),(4.15)$ becomes:

$$
\begin{aligned}
& \left\|\boldsymbol{e}_{h}^{n+1}\right\|_{\mathbf{L}^{2}}^{2}+2 \nu \Delta t\left\|D\left(\boldsymbol{e}_{h}^{n+1}\right)\right\|_{\mathbf{L}^{2}}^{2}+\Delta t\left\|\sigma_{h}^{*}\left(\nabla \lambda_{h}^{n+1}\right)\right\|_{\tau_{p}}^{2} \\
& \quad \leq \quad\left(1+C \nu^{-1} \Delta t\|\boldsymbol{u}\|_{L^{\infty}\left(\mathbf{H}^{s+1}\right)}^{2}\right)\left\|\boldsymbol{e}_{h}^{n}\right\|_{\mathbf{L}^{2}}^{2}+\nu^{-1} \Delta t\left\|\varepsilon_{v h}^{n+1}\right\|_{\mathbf{H}^{-1}}^{2}+C \Delta t h^{2 s}\|\boldsymbol{u}\|_{L^{\infty}\left(\mathbf{H}^{s+1}\right)}^{2} \\
& \quad+\Delta t\left[\left\|\sigma_{h}^{*}\left(\nabla \widehat{p}_{h}^{n+1}\right)\right\|_{\tau_{p}}^{2}+2 s_{\text {conv }}\left(\boldsymbol{u}_{h}^{n}, \boldsymbol{u}_{h}^{n+1}, \boldsymbol{e}_{h}^{n+1}\right)+2 s_{\operatorname{div}}\left(\boldsymbol{u}_{h}^{n+1}, \boldsymbol{e}_{h}^{n+1}\right)\right] .
\end{aligned}
$$

To bound $\left\|\sigma_{h}^{*}\left(\nabla \widehat{p}_{h}^{n+1}\right)\right\|_{\tau_{p}}$, we add and subtract $\nabla \widehat{p}_{h}^{n+1}$ and use Lemma 4.1 and the optimal error estimates (3.7) and (4.11):

$$
\begin{aligned}
\left\|\sigma_{h}^{*}\left(\nabla \widehat{p}_{h}^{n+1}\right)\right\|_{\tau_{p}} & \leq\left\|\sigma_{h}^{*}\left(\nabla \widehat{\lambda}_{h}^{n+1}\right)\right\|_{\tau_{p}}+\left\|\sigma_{h}^{*}\left(\nabla p^{n+1}\right)\right\|_{\tau_{p}} \\
& \leq C h\left\|\nabla \widehat{\lambda}_{h}^{n+1}\right\|_{\mathbf{L}^{2}}+C h\left\|\sigma_{h}^{*}\left(\nabla p^{n+1}\right)\right\|_{\mathbf{L}^{2}} \leq C h^{s}\|p\|_{L^{\infty}\left(H^{s}\right)} .
\end{aligned}
$$

Combining estimate (4.18) with (4.17), we obtain:

$$
\begin{aligned}
&\left\|\boldsymbol{e}_{h}^{n+1}\right\|_{\mathbf{L}^{2}}^{2}+2 \nu \Delta t\left\|D\left(\boldsymbol{e}_{h}^{n+1}\right)\right\|_{\mathbf{L}^{2}}^{2}+\Delta t\left\|\sigma_{h}^{*}\left(\nabla \lambda_{h}^{n+1}\right)\right\|_{\tau_{p}}^{2} \\
& \leq \quad\left(1+C \nu^{-1} \Delta t\|\boldsymbol{u}\|_{L^{\infty}\left(\mathbf{H}^{s+1}\right)}^{2}\right)\left\|\boldsymbol{e}_{h}^{n}\right\|_{\mathbf{L}^{2}}^{2}+\nu^{-1} \Delta t\left\|\varepsilon_{v h}^{n+1}\right\|_{\mathbf{H}^{-1}}^{2} \\
&+C \Delta t h^{2 s}\left(\|\boldsymbol{u}\|_{L^{\infty}\left(\mathbf{H}^{s+1}\right)}^{2}+\|p\|_{L^{\infty}\left(H^{s}\right)}^{2}\right) \\
&+2 \Delta t\left[s_{\operatorname{conv}}\left(\boldsymbol{u}_{h}^{n}, \boldsymbol{u}_{h}^{n+1}, \boldsymbol{e}_{h}^{n+1}\right)+s_{\operatorname{div}}\left(\boldsymbol{u}_{h}^{n+1}, \boldsymbol{e}_{h}^{n+1}\right)\right] .
\end{aligned}
$$

Arguing similarly for the forms $s_{\text {conv }}$ and $s_{\text {div }}$, we have:

$$
\begin{aligned}
s_{\operatorname{div}}\left(\boldsymbol{u}_{h}^{n+1}, \boldsymbol{e}_{h}^{n+1}\right) & \leq \frac{1}{2}\left(\left\|\sigma_{h}^{*}\left(\nabla \cdot \widehat{\boldsymbol{u}}_{h}^{n+1}\right)\right\|_{\tau_{d}}^{2}-\left\|\sigma_{h}^{*}\left(\nabla \cdot \boldsymbol{e}_{h}^{n+1}\right)\right\|_{\tau_{d}}^{2}\right) \\
& \leq C h^{2 s}\|\boldsymbol{u}\|_{L^{\infty}\left(\mathbf{H}^{s+1}\right)}^{2}-\frac{1}{2}\left\|\sigma_{h}^{*}\left(\nabla \cdot \boldsymbol{e}_{h}^{n+1}\right)\right\|_{\tau_{d}}^{2}, \\
s_{\mathrm{conv}}\left(\boldsymbol{u}_{h}^{n}, \boldsymbol{u}_{h}^{n+1}, \boldsymbol{e}_{h}^{n+1}\right) & \leq \frac{1}{2}\left(\left\|\sigma_{h}^{*}\left(\boldsymbol{u}_{h}^{n} \cdot \nabla \widehat{\boldsymbol{u}}_{h}^{n+1}\right)\right\|_{\tau_{\nu}}^{2}-\left\|\sigma_{h}^{*}\left(\boldsymbol{u}_{h}^{n} \cdot \nabla \boldsymbol{e}_{h}^{n+1}\right)\right\|_{\tau_{\nu}}^{2}\right) .
\end{aligned}
$$

Thus, from (4.19) we obtain:

$$
\begin{aligned}
\left\|\boldsymbol{e}_{h}^{n+1}\right\|_{\mathbf{L}^{2}}^{2}+2 \nu \Delta t\left\|D\left(\boldsymbol{e}_{h}^{n+1}\right)\right\|_{\mathbf{L}^{2}}^{2} & \\
& +\Delta t\left[\left\|\sigma_{h}^{*}\left(\boldsymbol{u}_{h}^{n} \cdot \nabla \boldsymbol{e}_{h}^{n+1}\right)\right\|_{\tau_{\nu}}^{2}+\left\|\sigma_{h}^{*}\left(\nabla \cdot \boldsymbol{e}_{h}^{n+1}\right)\right\|_{\tau_{d}}^{2}+\left\|\sigma_{h}^{*}\left(\nabla \lambda_{h}^{n+1}\right)\right\|_{\tau_{p}}^{2}\right] \\
\leq & \left(1+C \nu^{-1} \Delta t\|\boldsymbol{u}\|_{L^{\infty}\left(\mathbf{H}^{s+1}\right)}^{2}\right)\left\|\boldsymbol{e}_{h}^{n}\right\|_{\mathbf{L}^{2}}^{2} \\
(4.20) & +C \Delta t\left[h^{2 s}\left(\|\boldsymbol{u}\|_{L^{\infty}\left(\mathbf{H}^{s+1}\right)}^{2}+\|p\|_{L^{\infty}\left(H^{s}\right)}^{2}\right)+\nu^{-1}\left\|\varepsilon_{v h}^{n+1}\right\|_{\mathbf{H}^{-1}}^{2}+\left\|\sigma_{h}^{*}\left(\boldsymbol{u}_{h}^{n} \cdot \nabla \widehat{\boldsymbol{u}}_{h}^{n+1}\right)\right\|_{\tau_{\nu}}^{2}\right] .
\end{aligned}
$$

The estimate for $\left\|\sigma_{h}^{*}\left(\boldsymbol{u}_{h}^{n} \cdot \nabla \widehat{\boldsymbol{u}}_{h}^{n+1}\right)\right\|_{\tau_{\nu}}^{2}$ is rather involved, so that we discuss it in detail. By applying the triangle inequality and Lemma 4.1, we have:

$$
\begin{aligned}
& \left\|\sigma_{h}^{*}\left(\boldsymbol{u}_{h}^{n} \cdot \nabla \widehat{\boldsymbol{u}}_{h}^{n+1}\right)\right\|_{\tau_{\nu}} \\
& \quad \leq\left\|\sigma_{h}^{*}\left(\boldsymbol{u}_{h}^{n} \cdot \nabla \widehat{\boldsymbol{e}}_{h}^{n+1}\right)\right\|_{\tau_{\nu}}+\left\|\sigma_{h}^{*}\left(\boldsymbol{u}_{h}^{n} \cdot \nabla \boldsymbol{u}^{n+1}\right)\right\|_{\tau_{\nu}} \\
& \quad \leq C h\left\|\boldsymbol{u}_{h}^{n} \cdot \nabla \widehat{\boldsymbol{e}}_{h}^{n+1}\right\|_{\mathbf{L}^{2}}+\left\|\sigma_{h}^{*}\left(\boldsymbol{u}_{h}^{n} \cdot \nabla \boldsymbol{u}^{n+1}\right)\right\|_{\tau_{\nu}} \\
& \quad \leq C h\left(\left\|\boldsymbol{e}_{h}^{n} \cdot \nabla \widehat{\boldsymbol{e}}_{h}^{n+1}\right\|_{\mathbf{L}^{2}}+\left\|\widehat{\boldsymbol{u}}_{h}^{n} \cdot \nabla \widehat{\boldsymbol{e}}_{h}^{n+1}\right\|_{\mathbf{L}^{2}}\right)+\left\|\sigma_{h}^{*}\left(\boldsymbol{u}_{h}^{n} \cdot \nabla \boldsymbol{u}^{n+1}\right)\right\|_{\tau_{\nu}}
\end{aligned}
$$


The first two terms in the last inequality are bounded by using Hölder's inequality, (4.14), and the optimal error estimate (4.10):

$$
\begin{aligned}
h & \left(\left\|\boldsymbol{e}_{h}^{n} \cdot \nabla \widehat{\boldsymbol{e}}_{h}^{n+1}\right\|_{\mathbf{L}^{2}}+\left\|\widehat{\boldsymbol{u}}_{h}^{n} \cdot \nabla \widehat{\boldsymbol{e}}_{h}^{n+1}\right\|_{\mathbf{L}^{2}}\right) \\
& \leq h\left(\left\|\boldsymbol{e}_{h}^{n}\right\|_{\mathbf{L}^{2}}\left\|\nabla \widehat{\boldsymbol{e}}_{h}^{n+1}\right\|_{\mathbf{L}^{\infty}}+\left\|\widehat{\boldsymbol{u}}_{h}^{n}\right\|_{\mathbf{L}^{\infty}}\left\|\nabla \widehat{\boldsymbol{e}}_{h}^{n+1}\right\|_{\mathbf{L}^{2}}\right) \\
& \leq C h\|\boldsymbol{u}\|_{L^{\infty}\left(\mathbf{H}^{s+1}\right)}\left(\left\|\boldsymbol{e}_{h}^{n}\right\|_{\mathbf{L}^{2}} h^{s-d / 2}+\left\|\nabla \widehat{\boldsymbol{e}}_{h}^{n+1}\right\|_{\mathbf{L}^{2}}\right) \\
& \leq C\left(\|\boldsymbol{u}\|_{L^{\infty}\left(\mathbf{H}^{s+1}\right)}\left\|\boldsymbol{e}_{h}^{n}\right\|_{\mathbf{L}^{2}} h^{s+1-d / 2}+\|\boldsymbol{u}\|_{L^{\infty}\left(\mathbf{H}^{s+1}\right)}^{2} h^{s+1}\right) .
\end{aligned}
$$

The last term in (4.21) is bounded by using again Lemma 4.1, the stability estimate (3.6), the properties $\nabla \boldsymbol{u}^{n+1} \in \mathbf{L}^{\infty}$ and $\left(\boldsymbol{u}^{n} \cdot \nabla \boldsymbol{u}^{n+1}\right) \in \mathbf{H}^{s}$, which follow from the regularity assumptions and the Sobolev imbedding theorem, and the optimal error estimates (3.7) and (4.10):

$$
\begin{aligned}
&\left\|\sigma_{h}^{*}\left(\boldsymbol{u}_{h}^{n} \cdot \nabla \boldsymbol{u}^{n+1}\right)\right\|_{\tau_{\nu}} \leq C h\left\|\sigma_{h}^{*}\left(\boldsymbol{u}_{h}^{n} \cdot \nabla \boldsymbol{u}^{n+1}\right)\right\|_{\mathbf{L}^{2}} \\
& \leq C h\left(\left\|\sigma_{h}^{*}\left(\boldsymbol{e}_{h}^{n} \cdot \nabla \boldsymbol{u}^{n+1}\right)\right\|_{\mathbf{L}^{2}}+\left\|\sigma_{h}^{*}\left(\widehat{\boldsymbol{u}}_{h}^{n} \cdot \nabla \boldsymbol{u}^{n+1}\right)\right\|_{\mathbf{L}^{2}}\right) \\
& \leq C h\left(\left\|\boldsymbol{e}_{h}^{n} \cdot \nabla \boldsymbol{u}^{n+1}\right\|_{\mathbf{L}^{2}}+\left\|\widehat{\boldsymbol{e}}_{h}^{n} \cdot \nabla \boldsymbol{u}^{n+1}\right\|_{\mathbf{L}^{2}}+\left\|\sigma_{h}^{*}\left(\boldsymbol{u}^{n} \cdot \nabla \boldsymbol{u}^{n+1}\right)\right\|_{\mathbf{L}^{2}}\right) \\
&(4.23) \leq C h\|\boldsymbol{u}\|_{L^{\infty}\left(\mathbf{H}^{s+1}\right)}\left(\left\|\boldsymbol{e}_{h}^{n}\right\|_{\mathbf{L}^{2}}+h^{s}\|\boldsymbol{u}\|_{L^{\infty}\left(\mathbf{H}^{s+1}\right)}\right) .
\end{aligned}
$$

Combining (4.21) with (4.22) and (4.23), we finally obtain:

$$
\left\|\sigma_{h}^{*}\left(\boldsymbol{u}_{h}^{n} \cdot \nabla \widehat{\boldsymbol{u}}_{h}^{n+1}\right)\right\|_{\tau_{\nu}} \leq C\left(h\|\boldsymbol{u}\|_{L^{\infty}\left(\mathbf{H}^{s+1}\right)}\left\|\boldsymbol{e}_{h}^{n}\right\|_{\mathbf{L}^{2}}+h^{s+1}\|\boldsymbol{u}\|_{L^{\infty}\left(\mathbf{H}^{s+1}\right)}^{2}\right) .
$$

Inserting (4.24) into (4.20) gives in particular:

$$
\begin{aligned}
\left\|\boldsymbol{e}_{h}^{n+1}\right\|_{\mathbf{L}^{2}}^{2}+2 \nu \Delta t\left\|D\left(\boldsymbol{e}_{h}^{n+1}\right)\right\|_{\mathbf{L}^{2}}^{2} & \\
& +\Delta t\left[\left\|\sigma_{h}^{*}\left(\boldsymbol{u}_{h}^{n} \cdot \nabla \boldsymbol{e}_{h}^{n+1}\right)\right\|_{\tau_{\nu}}^{2}+\left\|\sigma_{h}^{*}\left(\nabla \cdot \boldsymbol{e}_{h}^{n+1}\right)\right\|_{\tau_{d}}^{2}+\left\|\sigma_{h}^{*}\left(\nabla \lambda_{h}^{n+1}\right)\right\|_{\tau_{p}}^{2}\right] \\
\leq & {\left[1+C\left(\nu^{-1}+h^{2}\right) \Delta t\|\boldsymbol{u}\|_{L^{\infty}\left(\mathbf{H}^{s+1}\right)}^{2}\right]\left\|\boldsymbol{e}_{h}^{n}\right\|_{\mathbf{L}^{2}}^{2}+C \nu^{-1}\left\|\varepsilon_{v h}^{n+1}\right\|_{\mathbf{H}^{-1}}^{2} } \\
& +C h^{2 s}\left(\|\boldsymbol{u}\|_{L^{\infty}\left(\mathbf{H}^{s+1}\right)}^{2}+\|p\|_{L^{\infty}\left(H^{s}\right)}^{2}+\|\boldsymbol{u}\|_{L^{\infty}\left(\mathbf{H}^{s+1}\right)}^{4}\right) \Delta t .
\end{aligned}
$$

We now apply the discrete Gronwall's lemma 4.5 with:

$$
\begin{aligned}
\alpha_{n} & =\left\|\boldsymbol{e}_{h}^{n}\right\|_{\mathbf{L}^{2}}^{2}, \\
\beta_{n} & =C\left[\nu^{-1}\left\|\varepsilon_{v h}^{n+1}\right\|_{\mathbf{H}^{-1}}^{2}+h^{2 s}\left(\|\boldsymbol{u}\|_{L^{\infty}\left(\mathbf{H}^{s+1}\right)}^{2}+\|p\|_{L^{\infty}\left(H^{s}\right)}^{2}+\|\boldsymbol{u}\|_{L^{\infty}\left(\mathbf{H}^{s+1}\right)}^{4}\right)\right] \Delta t, \\
C_{n} & =0, \quad D_{n}=C\left(\nu^{-1}+h^{2}\right)\|\boldsymbol{u}\|_{L^{\infty}\left(\mathbf{H}^{s+1}\right)}^{2},
\end{aligned}
$$

to deduce:

$$
\begin{aligned}
& \max _{n=0,1, \ldots, N}\left\|\boldsymbol{e}_{h}^{n}\right\|_{\mathbf{L}^{2}}^{2} \leq\left\|\boldsymbol{e}_{h}^{0}\right\|_{\mathbf{L}^{2}}^{2} e^{\widetilde{C}} \\
& \quad+C e^{\widetilde{C}} h^{2 s}\left(\|\boldsymbol{u}\|_{L^{\infty}\left(\mathbf{H}^{s+1}\right)}^{2}+\|p\|_{L^{\infty}\left(H^{s}\right)}^{2}+\|\boldsymbol{u}\|_{L^{\infty}\left(\mathbf{H}^{s+1}\right)}^{4}\right) T \\
& \quad+C e^{\widetilde{C}} \nu^{-1} \sum_{n=0}^{N-1} \Delta t\left\|\varepsilon_{v h}^{n+1}\right\|_{\mathbf{H}^{-1}}^{2}
\end{aligned}
$$

where $\widetilde{C}=C(T)\left(1+\nu^{-1}\right)\|\boldsymbol{u}\|_{L^{\infty}\left(\mathbf{H}^{s+1}\right)}^{2}$, and $C(T)>0$ is an increasing function of $T$, independent of $h$ and $\Delta t$. Summing (4.25) from $n=0$ to $n=r-1$ for a positive integer 
$r \leq N$ and using (4.26), we obtain:

$$
\begin{aligned}
\left\|\boldsymbol{e}_{h}^{r}\right\|_{\mathbf{L}^{2}}^{2} & +2 \nu \sum_{n=0}^{r-1} \Delta t\left\|D\left(\boldsymbol{e}_{h}^{n+1}\right)\right\|_{\mathbf{L}^{2}}^{2} \\
& +\sum_{n=0}^{r-1} \Delta t\left[\left\|\sigma_{h}^{*}\left(\boldsymbol{u}_{h}^{n} \cdot \nabla \boldsymbol{e}_{h}^{n+1}\right)\right\|_{\tau_{\nu}}^{2}+\left\|\sigma_{h}^{*}\left(\nabla \cdot \boldsymbol{e}_{h}^{n+1}\right)\right\|_{\tau_{d}}^{2}+\left\|\sigma_{h}^{*}\left(\nabla \lambda_{h}^{n+1}\right)\right\|_{\tau_{p}}^{2}\right] \\
\leq & \widetilde{C} \max _{n=0,1, \ldots, N}\left\|\boldsymbol{e}_{h}^{n}\right\|_{\mathbf{L}^{2}}^{2}+C \nu^{-1} \sum_{n=0}^{r-1} \Delta t\left\|\varepsilon_{v h}^{n+1}\right\|_{\mathbf{H}^{-1}}^{2} \\
& +C h^{2 s}\left(\|\boldsymbol{u}\|_{L^{\infty}\left(\mathbf{H}^{s+1}\right)}^{2}+\|p\|_{L^{\infty}\left(H^{s}\right)}^{2}+\|\boldsymbol{u}\|_{L^{\infty}\left(\mathbf{H}^{s+1}\right)}^{4}\right) T \\
\leq & \widetilde{C} e^{\widetilde{C}}\left(\left\|\boldsymbol{e}_{h}^{0}\right\|_{\mathbf{L}^{2}}^{2}+\nu^{-1} \sum_{n=0}^{N-1} \Delta t\left\|\varepsilon_{v h}^{n+1}\right\|_{\mathbf{H}^{-1}}^{2}\right) \\
& +\widetilde{C} e^{\widetilde{C}} h^{2 s}\left(\|\boldsymbol{u}\|_{L^{\infty}\left(\mathbf{H}^{s+1}\right)}^{2}+\|p\|_{L^{\infty}\left(H^{s}\right)}^{2}+\|\boldsymbol{u}\|_{L^{\infty}\left(\mathbf{H}^{s+1}\right)}^{4}\right) .
\end{aligned}
$$

Step 3: Consistency velocity error estimate. For the analysis, the consistency error is decomposed into $\left\langle\varepsilon_{v h}^{n+1}, \boldsymbol{v}\right\rangle=\sum_{i=1}^{5} \varepsilon_{i}$, with:

$$
\begin{aligned}
& \varepsilon_{1}=-\left(\partial_{t} \boldsymbol{u}^{n+1}-\frac{\widehat{\boldsymbol{u}}_{h}^{n+1}-\widehat{\boldsymbol{u}}_{h}^{n}}{\Delta t}, \boldsymbol{v}\right)_{\Omega}, \quad \varepsilon_{2}=b\left(\widehat{\boldsymbol{u}}_{h}^{n}, \widehat{\boldsymbol{u}}_{h}^{n+1}, \boldsymbol{v}\right)-b\left(\boldsymbol{u}^{n+1} ; \boldsymbol{u}^{n+1}, \boldsymbol{v}\right), \\
& \varepsilon_{3}=a\left(\widehat{\boldsymbol{e}}_{h}^{n+1}, \boldsymbol{v}\right), \quad \varepsilon_{4}=-\left(\widehat{\lambda}_{h}^{n+1}, \nabla \cdot \boldsymbol{v}\right)_{\Omega}, \quad \varepsilon_{5}=\left\langle\boldsymbol{f}^{n+1}-\overline{\boldsymbol{f}}^{n+1}, \boldsymbol{v}\right\rangle,
\end{aligned}
$$

where we recall that $\widehat{\boldsymbol{e}}_{h}^{n+1}=\widehat{\boldsymbol{u}}_{h}^{n+1}-\boldsymbol{u}^{n+1}$ and $\widehat{\lambda}_{h}^{n+1}=\widehat{p}_{h}^{n+1}-p^{n+1}$. Estimates for $\varepsilon_{i}$, $i=1, \ldots, 5$, can be directly derived from [21], pages $380-381$, thus we skip them for brevity. Collecting all these estimates yields:

$$
\begin{aligned}
\left\|\varepsilon_{v h}^{n+1}\right\|_{\mathbf{H}^{-1}}^{2}= & \left(\sup _{\boldsymbol{v} \in \mathbf{H}_{0}^{1}} \frac{\left\langle\varepsilon_{v h}^{n+1}, \boldsymbol{v}\right\rangle}{\|D(\boldsymbol{v})\|_{\mathbf{L}^{2}}}\right)^{2} \leq C(\Delta t)^{-2} h^{2(s+1)}\|\boldsymbol{u}\|_{L^{\infty}\left(\mathbf{H}^{s+1}\right)}^{2} \\
& +C h^{2 s}\left(\|\boldsymbol{u}\|_{L^{\infty}\left(\mathbf{H}^{s+1}\right)}^{2}+\|p\|_{L^{\infty}\left(H^{s}\right)}^{2}\right) \\
& +C \Delta t \int_{t_{n}}^{t_{n+1}}\left(\left\|\partial_{s s} \boldsymbol{u}(s)\right\|_{\mathbf{L}^{2}}^{2}+\left\|\partial_{s} \boldsymbol{u}(s)\right\|_{\mathbf{L}^{2}}^{2}+\left\|\partial_{s} \boldsymbol{f}(s)\right\|_{\mathbf{H}^{-1}}^{2}\right) d s .
\end{aligned}
$$

Summation over the discrete times and using the regularity assumptions on $\boldsymbol{u}, p$ and $\boldsymbol{f}$ (the norms concerning the time derivatives of $\boldsymbol{u}$ and $\boldsymbol{f}$ are hidden in the constant) leads to:

$$
\begin{aligned}
\sum_{n=0}^{N-1} \Delta t\left\|\varepsilon_{v h}^{n+1}\right\|_{\mathbf{H}^{-1}}^{2} \leq & C(\Delta t)^{-2} h^{2(s+1)}\|\boldsymbol{u}\|_{L^{\infty}\left(\mathbf{H}^{s+1}\right)}^{2} \\
& +C h^{2 s}\left(\|\boldsymbol{u}\|_{L^{\infty}\left(\mathbf{H}^{s+1}\right)}^{2}+\|p\|_{L^{\infty}\left(H^{s}\right)}^{2}\right) \\
& +C(\Delta t)^{2}\left[\left\|\partial_{t t} \boldsymbol{u}\right\|_{L^{2}\left(\mathbf{L}^{2}\right)}^{2}+\left\|\partial_{t} \boldsymbol{u}\right\|_{L^{2}\left(\mathbf{L}^{2}\right)}^{2}+\left\|\partial_{t} \boldsymbol{f}\right\|_{L^{2}\left(\mathbf{H}^{-1}\right)}^{2}\right] \\
\leq & C\left[(\Delta t)^{-2} h^{2(s+1)}\|\boldsymbol{u}\|_{L^{\infty}\left(\mathbf{H}^{s+1}\right)}^{2}+(\Delta t)^{2}\right] \\
& +C h^{2 s}\left(\|\boldsymbol{u}\|_{L^{\infty}\left(\mathbf{H}^{s+1}\right)}^{2}+\|p\|_{L^{\infty}\left(H^{s}\right)}^{2}\right) .
\end{aligned}
$$


Combining (4.27) with (4.28), we obtain, for $0<r \leq N$ :

$$
\begin{aligned}
\left\|\boldsymbol{e}_{h}^{r}\right\|_{\mathbf{L}^{2}}^{2} & +2 \nu \sum_{n=0}^{r-1} \Delta t\left\|D\left(\boldsymbol{e}_{h}^{n+1}\right)\right\|_{\mathbf{L}^{2}}^{2} \\
& +\sum_{n=0}^{r-1} \Delta t\left[\left\|\sigma_{h}^{*}\left(\boldsymbol{u}_{h}^{n} \cdot \nabla \boldsymbol{e}_{h}^{n+1}\right)\right\|_{\tau_{\nu}}^{2}+\left\|\sigma_{h}^{*}\left(\nabla \cdot \boldsymbol{e}_{h}^{n+1}\right)\right\|_{\tau_{d}}^{2}+\left\|\sigma_{h}^{*}\left(\nabla \lambda_{h}^{n+1}\right)\right\|_{\tau_{p}}^{2}\right] \\
\leq & \widetilde{C} e^{\widetilde{C}}\left[\left\|\boldsymbol{e}_{h}^{0}\right\|_{\mathbf{L}^{2}}^{2}+h^{2 s}\left(\|\boldsymbol{u}\|_{L^{\infty}\left(\mathbf{H}^{s+1}\right)}^{2}+\|p\|_{L^{\infty}\left(H^{s}\right)}^{2}+\|\boldsymbol{u}\|_{L^{\infty}\left(\mathbf{H}^{s+1}\right)}^{4}\right)\right] \\
& +\widetilde{C} e^{\widetilde{C}}\left[(\Delta t)^{-2} h^{2(s+1)}\|\boldsymbol{u}\|_{L^{\infty}\left(\mathbf{H}^{s+1}\right)}^{2}+(\Delta t)^{2}\right],
\end{aligned}
$$

where we recall that $\widetilde{C}=C(T)\left(1+\nu^{-1}\right)\|\boldsymbol{u}\|_{\mathbf{H}^{s+1}}^{2}$, and $C(T)>0$ is an increasing function of $T$. With the notations of Theorem 4.6, we can write:

$$
\begin{aligned}
& \left\|\boldsymbol{e}_{h}\right\|_{\ell^{\infty}\left(\mathbf{L}^{2}\right)}+\sqrt{\nu}\left\|D\left(\boldsymbol{e}_{h}\right)\right\|_{\ell^{2}\left(\mathbf{L}^{2}\right)}=\max _{n=1, \ldots, N}\left\|\boldsymbol{e}_{h}^{n}\right\|_{\mathbf{L}^{2}}+\sqrt{\nu}\left[\sum_{n=1}^{N} \Delta t\left\|D\left(\boldsymbol{e}_{h}^{n}\right)\right\|_{\mathbf{L}^{2}}^{2}\right]^{1 / 2} \\
& \leq \sqrt{\widetilde{C} e^{\widetilde{C}}}\left[h^{s}\left(\|\boldsymbol{u}\|_{L^{\infty}\left(\mathbf{H}^{s+1}\right)}+\|p\|_{L^{\infty}\left(H^{s}\right)}+\|\boldsymbol{u}\|_{L^{\infty}\left(\mathbf{H}^{s+1}\right)}^{2}+1\right)\right] \\
& \quad+\sqrt{\widetilde{C} e^{\widetilde{C}}}\left[(\Delta t)^{-1} h^{s+1}\|\boldsymbol{u}\|_{L^{\infty}\left(\mathbf{H}^{s+1}\right)}+\Delta t\right] \\
& \leq \sqrt{\widetilde{C} e^{\widetilde{C}}}\left[h^{s}\left(\|\boldsymbol{u}\|_{L^{\infty}\left(\mathbf{H}^{s+1}\right)}+\|p\|_{L^{\infty}\left(H^{s}\right)}+\|\boldsymbol{u}\|_{L^{\infty}\left(\mathbf{H}^{s+1}\right)}^{2}+1\right)+\Delta t\right]
\end{aligned}
$$

using the assumption $C h \leq \Delta t$. Estimate (4.8) for the velocity follows from (4.30) using the triangle inequality:

$$
\begin{aligned}
\left\|\boldsymbol{u}-\boldsymbol{u}_{h}\right\|_{\ell^{\infty}\left(\mathbf{L}^{2}\right)}+\sqrt{\nu}\left\|D\left(\boldsymbol{u}-\boldsymbol{u}_{h}\right)\right\|_{\ell^{2}\left(\mathbf{L}^{2}\right) \leq} & \left\|\boldsymbol{e}_{h}\right\|_{\ell^{\infty}\left(\mathbf{L}^{2}\right)}+\sqrt{\nu}\left\|D\left(\boldsymbol{e}_{h}\right)\right\|_{\ell^{2}\left(\mathbf{L}^{2}\right)} \\
& +\left\|\widehat{\boldsymbol{e}}_{h}\right\|_{\ell^{\infty}\left(\mathbf{L}^{2}\right)}+\sqrt{\nu}\left\|D\left(\widehat{\boldsymbol{e}}_{h}\right)\right\|_{\ell^{2}\left(\mathbf{L}^{2}\right)},
\end{aligned}
$$

and the optimal error estimate (4.10).

Step 4: Pressure estimate. From the error equation (4.13), setting $q_{h}=0$, we have:

$$
\begin{aligned}
\left(\lambda_{h}^{n+1}, \nabla \cdot \boldsymbol{v}_{h}\right)_{\Omega}= & \left(\frac{\boldsymbol{e}_{h}^{n+1}-\boldsymbol{e}_{h}^{n}}{\Delta t}, \boldsymbol{v}_{h}\right)_{\Omega}+b\left(\boldsymbol{u}_{h}^{n}, \boldsymbol{e}_{h}^{n+1}, \boldsymbol{v}_{h}\right)+b\left(\boldsymbol{e}_{h}^{n}, \widehat{\boldsymbol{u}}_{h}^{n+1}, \boldsymbol{v}_{h}\right) \\
& +a\left(\boldsymbol{e}_{h}^{n+1}, \boldsymbol{v}_{h}\right)-\left\langle\varepsilon_{v h}^{n+1}, \boldsymbol{v}_{h}\right\rangle-s_{\mathrm{conv}}\left(\boldsymbol{u}_{h}^{n}, \boldsymbol{u}_{h}^{n+1}, \boldsymbol{v}_{h}\right)-s_{\mathrm{div}}\left(\boldsymbol{u}_{h}^{n+1}, \boldsymbol{v}_{h}\right) .
\end{aligned}
$$

Let $\Lambda_{h}^{n+1}=\sum_{k=0}^{n} \Delta t \lambda_{h}^{k+1}=\sum_{k=0}^{n} \Delta t\left(\widehat{p}_{h}^{k+1}-p_{h}^{k+1}\right)$, then summation over the discrete times gives:

$$
\begin{aligned}
& \left(\Lambda_{h}^{n+1}, \nabla \cdot \boldsymbol{v}_{h}\right)_{\Omega} \\
& =\quad\left(\boldsymbol{e}_{h}^{n+1}-\boldsymbol{e}_{h}^{0}, \boldsymbol{v}_{h}\right)_{\Omega}+\sum_{k=0}^{n} \Delta t\left[b\left(\boldsymbol{u}_{h}^{k}, \boldsymbol{e}_{h}^{k+1}, \boldsymbol{v}_{h}\right)+b\left(\boldsymbol{e}_{h}^{k}, \widehat{\boldsymbol{u}}_{h}^{k+1}, \boldsymbol{v}_{h}\right)\right] \\
& \quad+\sum_{k=0}^{n} \Delta t\left[a\left(\boldsymbol{e}_{h}^{k+1}, \boldsymbol{v}_{h}\right)-\left\langle\varepsilon_{v h}^{k+1}, \boldsymbol{v}_{h}\right\rangle-s_{\mathrm{conv}}\left(\boldsymbol{u}_{h}^{k}, \boldsymbol{u}_{h}^{k+1}, \boldsymbol{v}_{h}\right)-s_{\operatorname{div}}\left(\boldsymbol{u}_{h}^{k+1}, \boldsymbol{v}_{h}\right)\right] .
\end{aligned}
$$

The application of the triangle inequality, a standard estimate for the convective term, the Cauchy-Schwarz and Korn's inequalities, the stability result (4.6) for the velocity, the 
regularity assumptions on $\boldsymbol{u}$, and the optimal error estimate (4.10) yield:

$$
\begin{aligned}
\frac{\left(\Lambda_{h}^{n+1}, \nabla \cdot \boldsymbol{v}_{h}\right)_{\Omega}}{\left\|D\left(\boldsymbol{v}_{h}\right)\right\|_{\mathbf{L}^{2}}} & {\left[\left(\left\|\boldsymbol{e}_{h}^{n+1}\right\|_{\mathbf{L}^{2}}+\left\|\boldsymbol{e}_{h}^{0}\right\|_{\mathbf{L}^{2}}\right)\right.} \\
\leq \quad & {\left[\sum_{k=0}^{n} \Delta t\left(\left\|D\left(\boldsymbol{u}_{h}^{k}\right)\right\|_{\mathbf{L}^{2}}\left\|D\left(\boldsymbol{e}_{h}^{k+1}\right)\right\|_{\mathbf{L}^{2}}+\left(\left\|D\left(\widehat{\boldsymbol{e}}_{h}^{k+1}\right)\right\|_{\mathbf{L}^{2}}+\left\|D\left(\boldsymbol{u}^{k+1}\right)\right\|_{\mathbf{L}^{2}}\right)\left\|D\left(\boldsymbol{e}_{h}^{k}\right)\right\|_{\mathbf{L}^{2}}\right)\right.} \\
+ & \sum_{k=0}^{n} \Delta t\left(\left\|D\left(\boldsymbol{e}_{h}^{k+1}\right)\right\|_{\mathbf{L}^{2}}+\left\|\boldsymbol{\varepsilon}_{v h}^{k+1}\right\|_{\mathbf{H}^{-1}}\right) \\
+ & \left.\frac{1}{\left\|D\left(\boldsymbol{v}_{h}\right)\right\|_{\mathbf{L}^{2}}} \sum_{k=0}^{n} \Delta t\left(\left|s_{\mathrm{conv}}\left(\boldsymbol{u}_{h}^{k}, \boldsymbol{u}_{h}^{k+1}, \boldsymbol{v}_{h}\right)\right|+\left|s_{\mathrm{div}}\left(\boldsymbol{u}_{h}^{k+1}, \boldsymbol{v}_{h}\right)\right|\right)\right] \\
\leq & {\left[\left(\left\|\boldsymbol{e}_{h}^{n+1}\right\|_{\mathbf{L}^{2}}+\left\|\boldsymbol{e}_{h}^{0}\right\|_{\mathbf{L}^{2}}+h^{s}\|\boldsymbol{u}\|_{L^{\infty}\left(\mathbf{H}^{s+1}\right)}\right)\right.} \\
+ & \left(\left(\sum_{k=0}^{n} \Delta t\left\|D\left(\boldsymbol{e}_{h}^{k+1}\right)\right\|_{\mathbf{L}^{2}}^{2}\right)^{1 / 2}+\left(\sum_{k=0}^{n} \Delta t\left\|\varepsilon_{v h}^{k+1}\right\|_{\mathbf{H}^{-1}}^{2}\right)^{1 / 2}\right) \\
(4.31)+ & \left.\frac{1}{\left\|D\left(\boldsymbol{v}_{h}\right)\right\|_{\mathbf{L}^{2}}} \sum_{k=0}^{n} \Delta t\left(\left|s_{\mathrm{conv}}\left(\boldsymbol{u}_{h}^{k}, \boldsymbol{u}_{h}^{k+1}, \boldsymbol{v}_{h}\right)\right|+\left|s_{\mathrm{div}}\left(\boldsymbol{u}_{h}^{k+1}, \boldsymbol{v}_{h}\right)\right|\right)\right]
\end{aligned}
$$

The stabilization term with respect to the divergence is bounded by the triangle inequality, the Cauchy-Schwarz inequality, (4.4), Poincaré's and Korn's inequalities, $\nabla \cdot \boldsymbol{u}^{k+1}=0$ a.e. in $\Omega$, the stability estimate (3.6), and the optimal error estimate (4.10):

$$
\begin{aligned}
\left|s_{\operatorname{div}}\left(\boldsymbol{u}_{h}^{k+1}, \boldsymbol{v}_{h}\right)\right| \leq & \left|s_{\operatorname{div}}\left(\widehat{\boldsymbol{e}}_{h}^{k+1}, \boldsymbol{v}_{h}\right)\right|+\left|s_{\operatorname{div}}\left(\boldsymbol{u}^{k+1}, \boldsymbol{v}_{h}\right)\right|+\left|s_{\operatorname{div}}\left(\boldsymbol{e}_{h}^{k+1}, \boldsymbol{v}_{h}\right)\right| \\
\leq & C\left(\left\|D\left(\widehat{\boldsymbol{e}}_{h}^{k+1}\right)\right\|_{\mathbf{L}^{2}}+\left\|\nabla \cdot \boldsymbol{u}^{k+1}\right\|_{\mathbf{L}^{2}}\right)\left\|D\left(\boldsymbol{v}_{h}\right)\right\|_{\mathbf{L}^{2}} \\
& +C\left\|D\left(\boldsymbol{e}_{h}^{k+1}\right)\right\|_{\mathbf{L}^{2}}\left\|D\left(\boldsymbol{v}_{h}\right)\right\|_{\mathbf{L}^{2}} \\
\leq & C\left(h^{s}\|\boldsymbol{u}\|_{L^{\infty}\left(\mathbf{H}^{s+1}\right)}+\left\|D\left(\boldsymbol{e}_{h}^{k+1}\right)\right\|_{\mathbf{L}^{2}}\right)\left\|D\left(\boldsymbol{v}_{h}\right)\right\|_{\mathbf{L}^{2}} .
\end{aligned}
$$

Using the triangle inequality, Lemma 3.4 in [19], and (4.10) yields:

$$
\begin{aligned}
\mid s_{\mathrm{conv}} & \left(\boldsymbol{u}_{h}^{k}, \boldsymbol{u}_{h}^{k+1}, \boldsymbol{v}_{h}\right) \mid \\
\leq & \left|s_{\mathrm{conv}}\left(\boldsymbol{u}_{h}^{k}, \widehat{\boldsymbol{e}}_{h}^{k+1}, \boldsymbol{v}_{h}\right)\right|+\left|s_{\mathrm{conv}}\left(\boldsymbol{u}_{h}^{k}, \boldsymbol{u}^{k+1}, \boldsymbol{v}_{h}\right)\right|+\left|s_{\mathrm{conv}}\left(\boldsymbol{u}_{h}^{k}, \boldsymbol{e}_{h}^{k+1}, \boldsymbol{v}_{h}\right)\right| \\
\leq & C h^{2+s-d / 2}\|\boldsymbol{u}\|_{L^{\infty}\left(\mathbf{H}^{s+1}\right)}\left\|D\left(\boldsymbol{u}_{h}^{k}\right)\right\|_{\mathbf{L}^{2}}^{2}\left\|D\left(\boldsymbol{v}_{h}\right)\right\|_{\mathbf{L}^{2}}+\left|s_{\mathrm{conv}}\left(\boldsymbol{u}_{h}^{k}, \boldsymbol{u}^{k+1}, \boldsymbol{v}_{h}\right)\right| \\
\quad & +\left|s_{\mathrm{conv}}\left(\boldsymbol{u}_{h}^{k}, \boldsymbol{e}_{h}^{k+1}, \boldsymbol{v}_{h}\right)\right| .
\end{aligned}
$$

Again, the triangle inequality, the application of Lemma 4.1, local inverse estimates ( $c f$. [8]), Sobolev injections, the stability estimate (3.6), and the optimal error approximation 
properties (3.7), (4.10) gives:

$$
\begin{aligned}
\left|s_{\text {conv }}\left(\boldsymbol{u}_{h}^{k}, \boldsymbol{u}^{k+1}, \boldsymbol{v}_{h}\right)\right| \leq & C \sum_{K \in \mathcal{T}_{h}} h_{K}^{2}\left\|\boldsymbol{e}_{h}^{k} \cdot \nabla \boldsymbol{u}^{k+1}\right\|_{L^{2}\left(\omega_{K}\right)}\left\|\boldsymbol{u}_{h}^{k} \cdot \nabla \boldsymbol{v}_{h}\right\|_{L^{2}\left(\omega_{K}\right)} \\
& +C \sum_{K \in \mathcal{T}_{h}} h_{K}^{2}\left\|\widehat{\boldsymbol{e}}_{h}^{k} \cdot \nabla \boldsymbol{u}^{k+1}\right\|_{L^{2}\left(\omega_{K}\right)}\left\|\boldsymbol{u}_{h}^{k} \cdot \nabla \boldsymbol{v}_{h}\right\|_{L^{2}\left(\omega_{K}\right)} \\
& +C \sum_{K \in \mathcal{T}_{h}} h_{K}^{2}\left\|\sigma_{h}^{*}\left(\boldsymbol{u}^{k} \cdot \nabla \boldsymbol{u}^{k+1}\right)\right\|_{L^{2}(K)}\left\|\boldsymbol{u}_{h}^{k} \cdot \nabla \boldsymbol{v}_{h}\right\|_{L^{2}\left(\omega_{K}\right)} \\
\leq & C h^{2}\left\|D\left(\boldsymbol{e}_{h}^{k}\right)\right\|_{\mathbf{L}^{2}}\left\|\nabla \boldsymbol{u}^{k+1}\right\|_{\mathbf{L}^{4}} h^{-d / 4}\left\|D\left(\boldsymbol{u}_{h}^{k}\right)\right\|_{\mathbf{L}^{2}}\left\|D\left(\boldsymbol{v}_{h}\right)\right\|_{\mathbf{L}^{2}} \\
& +C h^{2}\left\|D\left(\widehat{\boldsymbol{e}}_{h}^{k}\right)\right\|_{\mathbf{L}^{2}}\left\|\nabla \boldsymbol{u}^{k+1}\right\|_{\mathbf{L}^{4}} h^{-d / 4}\left\|D\left(\boldsymbol{u}_{h}^{k}\right)\right\|_{\mathbf{L}^{2}}\left\|D\left(\boldsymbol{v}_{h}\right)\right\|_{\mathbf{L}^{2}} \\
& +C h^{2}\left\|\sigma_{h}^{*}\left(\boldsymbol{u}^{k} \cdot \nabla \boldsymbol{u}^{k+1}\right)\right\|_{L^{2}} h^{-d / 4}\left\|D\left(\boldsymbol{u}_{h}^{k}\right)\right\|_{\mathbf{L}^{2}}\left\|D\left(\boldsymbol{v}_{h}\right)\right\|_{\mathbf{L}^{2}} \\
\leq & C h^{2-d / 4}\left\|D\left(\boldsymbol{e}_{h}^{k}\right)\right\|_{\mathbf{L}^{2}}\|\boldsymbol{u}\|_{L^{\infty}\left(\mathbf{H}^{s+1}\right)}\left\|D\left(\boldsymbol{u}_{h}^{k}\right)\right\|_{\mathbf{L}^{2}}\left\|D\left(\boldsymbol{v}_{h}\right)\right\|_{\mathbf{L}^{2}} \\
& +C h^{2-d / 4+s}\|\boldsymbol{u}\|_{L^{\infty}\left(\mathbf{H}^{s+1}\right)}^{2}\left\|D\left(\boldsymbol{u}_{h}^{k}\right)\right\|_{\mathbf{L}^{2}}\left\|D\left(\boldsymbol{v}_{h}\right)\right\|_{\mathbf{L}^{2}} .
\end{aligned}
$$

Moreover, by using Cauchy-Schwarz inequality and the stability property (3.6), we estimate:

$$
\begin{aligned}
\left|s_{\text {conv }}\left(\boldsymbol{u}_{h}^{k}, \boldsymbol{e}_{h}^{k+1}, \boldsymbol{v}_{h}\right)\right| & \leq C \sum_{K \in \mathcal{T}_{h}} \tau_{\nu, K}\left\|\sigma_{h}^{*}\left(\boldsymbol{u}_{h}^{k} \cdot \nabla \boldsymbol{e}_{h}^{k+1}\right)\right\|_{L^{2}(K)}\left\|\sigma_{h}^{*}\left(\boldsymbol{u}_{h}^{k} \cdot \nabla \boldsymbol{v}_{h}\right)\right\|_{L^{2}(K)} \\
& \leq C\left\|\sigma_{h}^{*}\left(\boldsymbol{u}_{h}^{k} \cdot \nabla \boldsymbol{e}_{h}^{k+1}\right)\right\|_{\tau_{\nu}}\left(\sum_{K \in \mathcal{T}_{h}} h_{K}^{2}\left\|\boldsymbol{u}_{h}^{k} \cdot \nabla \boldsymbol{v}_{h}\right\|_{L^{2}\left(\omega_{K}\right)}^{2}\right)^{1 / 2} \\
& \leq C\left\|\sigma_{h}^{*}\left(\boldsymbol{u}_{h}^{k} \cdot \nabla \boldsymbol{e}_{h}^{k+1}\right)\right\|_{\tau_{\nu}} h^{1-d / 4}\left\|D\left(\boldsymbol{u}_{h}^{k}\right)\right\|_{\mathbf{L}^{2}}\left\|D\left(\boldsymbol{v}_{h}\right)\right\|_{\mathbf{L}^{2}},
\end{aligned}
$$

where we have used local inverse estimates and Sobolev injections in the last inequality. Combining (4.34) and (4.35), from (4.33) we obtain:

$$
\begin{aligned}
\left|s_{\text {conv }}\left(\boldsymbol{u}_{h}^{k}, \boldsymbol{u}_{h}^{k+1}, \boldsymbol{v}_{h}\right)\right| \leq & C h^{2+s-d / 2}\|\boldsymbol{u}\|_{L^{\infty}\left(\mathbf{H}^{s+1}\right)}\left\|D\left(\boldsymbol{u}_{h}^{k}\right)\right\|_{\mathbf{L}^{2}}^{2}\left\|D\left(\boldsymbol{v}_{h}\right)\right\|_{\mathbf{L}^{2}} \\
& +C h^{2-d / 4}\left\|D\left(\boldsymbol{e}_{h}^{k}\right)\right\|_{\mathbf{L}^{2}}\|\boldsymbol{u}\|_{L^{\infty}\left(\mathbf{H}^{s+1}\right)}\left\|D\left(\boldsymbol{u}_{h}^{k}\right)\right\|_{\mathbf{L}^{2}}\left\|D\left(\boldsymbol{v}_{h}\right)\right\|_{\mathbf{L}^{2}} \\
& +C h^{2-d / 4+s}\|\boldsymbol{u}\|_{L^{\infty}\left(\mathbf{H}^{s+1}\right)}^{2}\left\|D\left(\boldsymbol{u}_{h}^{k}\right)\right\|_{\mathbf{L}^{2}}\left\|D\left(\boldsymbol{v}_{h}\right)\right\|_{\mathbf{L}^{2}} \\
& +C h^{1-d / 4}\left\|\sigma_{h}^{*}\left(\boldsymbol{u}_{h}^{k} \cdot \nabla \boldsymbol{e}_{h}^{k+1}\right)\right\|_{\tau_{\nu}}\left\|D\left(\boldsymbol{u}_{h}^{k}\right)\right\|_{\mathbf{L}^{2}}\left\|D\left(\boldsymbol{v}_{h}\right)\right\|_{\mathbf{L}^{2}} .
\end{aligned}
$$

Inserting (4.32) and (4.36) in (4.31), using the Cauchy-Schwarz inequality, the stability result (4.6) for the velocity, and taking advantage of estimate (4.29) to bound the last term in (4.36), we finally get:

$$
\begin{aligned}
\frac{\left(\Lambda_{h}^{n+1}, \nabla \cdot \boldsymbol{v}_{h}\right)_{\Omega}}{\left\|D\left(\boldsymbol{v}_{h}\right)\right\|_{\mathbf{L}^{2}}} \leq & \sqrt{\widetilde{C} e^{\widetilde{C}}}\left[\left\|\boldsymbol{e}_{h}^{n+1}\right\|_{\mathbf{L}^{2}}+\left\|\boldsymbol{e}_{h}^{0}\right\|_{\mathbf{L}^{2}}\right. \\
& +h^{s}\left(\|\boldsymbol{u}\|_{L^{\infty}\left(\mathbf{H}^{s+1}\right)}+\|p\|_{L^{\infty}\left(H^{s}\right)}+\|\boldsymbol{u}\|_{L^{\infty}\left(\mathbf{H}^{s+1}\right)}^{2}\right)+\Delta t \\
& \left.+\left(\sum_{k=0}^{n} \Delta t\left\|D\left(\boldsymbol{e}_{h}^{k+1}\right)\right\|_{\mathbf{L}^{2}}^{2}\right)^{1 / 2}+\left(\sum_{k=0}^{n} \Delta t\left\|\boldsymbol{\varepsilon}_{v h}^{k+1}\right\|_{\mathbf{H}^{-1}}^{2}\right)^{1 / 2}\right] .
\end{aligned}
$$


Thus, by the discrete inf-sup condition (4.5) and (4.28)-(4.29) it follows that:

$$
\begin{aligned}
\left\|\Lambda_{h}^{n+1}\right\|_{L^{2}} \leq & \sqrt{\widetilde{C} e^{\widetilde{C}}}\left[\sup _{k=0,1, \ldots, N}\left\|\boldsymbol{e}_{h}^{k}\right\|_{\mathbf{L}^{2}}+\left(\sum_{k=0}^{N-1} \Delta t\left\|D\left(\boldsymbol{e}_{h}^{k+1}\right)\right\|_{\mathbf{L}^{2}}^{2}\right)^{1 / 2}\right. \\
& +\left(\sum_{k=0}^{N-1} \Delta t\left\|\varepsilon_{v h}^{k+1}\right\|_{\mathbf{H}^{-1}}^{2}\right)^{1 / 2}+\left\|\sigma_{h}^{*}\left(\nabla \Lambda_{h}^{n+1}\right)\right\|_{\tau_{p}} \\
& \left.+h^{s}\left(\|\boldsymbol{u}\|_{L^{\infty}\left(\mathbf{H}^{s+1}\right)}+\|p\|_{L^{\infty}\left(H^{s}\right)}+\|\boldsymbol{u}\|_{L^{\infty}\left(\mathbf{H}^{s+1}\right)}^{2}+1\right)+\Delta t\right] \\
\leq & \sqrt{\widetilde{C} e^{\widetilde{C}}}\left[h^{s}\left(\|\boldsymbol{u}\|_{L^{\infty}\left(\mathbf{H}^{s+1}\right)}+\|p\|_{L^{\infty}\left(H^{s}\right)}+\|\boldsymbol{u}\|_{L^{\infty}\left(\mathbf{H}^{s+1}\right)}^{2}+1\right)+\Delta t\right] .
\end{aligned}
$$

Again, using the notation introduced in the statement of Theorem 4.6, in particular we can write:

$$
\begin{aligned}
& \left\|\Lambda_{h}\right\|_{\ell^{\infty}\left(L^{2}\right)}=\max _{n=1, \ldots, N}\left\|\Lambda_{h}^{n}\right\|_{L^{2}} \\
& \leq \sqrt{\widetilde{C} e^{\widetilde{C}}}\left[h^{s}\left(\|\boldsymbol{u}\|_{L^{\infty}\left(\mathbf{H}^{s+1}\right)}+\|p\|_{L^{\infty}\left(H^{s}\right)}+\|\boldsymbol{u}\|_{L^{\infty}\left(\mathbf{H}^{s+1}\right)}^{2}+1\right)+\Delta t\right] .
\end{aligned}
$$

Estimate (4.8) for the pressure follows from (4.39), the triangle inequality:

$$
\left\|\widetilde{P}-P_{h}\right\|_{\ell \infty\left(L^{2}\right)} \leq\left\|\Lambda_{h}\right\|_{\ell \infty\left(L^{2}\right)}+\left\|\widehat{\Lambda}_{h}\right\|_{\ell \infty\left(L^{2}\right)},
$$

and the optimal error estimate (4.11). This concludes the proof.

Remark 4.7. To obtain optimal estimates with respect to the polynomial interpolation, we must take $s=l$. This yields:

$$
\begin{aligned}
& \left\|\boldsymbol{u}-\boldsymbol{u}_{h}\right\|_{L^{\infty}\left(\mathbf{L}^{2}\right)}+\sqrt{\nu}\left\|D\left(\boldsymbol{u}-\boldsymbol{u}_{h}\right)\right\|_{L^{2}\left(\mathbf{L}^{2}\right)}+\left\|P-P_{h}\right\|_{L^{\infty}\left(L^{2}\right)} \\
& \quad \leq \sqrt{\widetilde{C} e^{\widetilde{C}}}\left[h^{l}\left(\|\boldsymbol{u}\|_{L^{\infty}\left(\mathbf{H}^{l+1}\right)}+\|p\|_{L^{\infty}\left(H^{l}\right)}+\|\boldsymbol{u}\|_{L^{\infty}\left(\mathbf{H}^{l+1}\right)}^{2}+1\right)+\Delta t\right] .
\end{aligned}
$$

Thus, the convergence of STAB method (3.2) is optimal with respect to the polynomial interpolation for smooth flows.

Remark 4.8. The proof of Theorem 4.6, that implies more concretely a strong convergence result for solutions with slightly increased regularity (it is sufficient $(\boldsymbol{u}, p) \in C^{0}\left(\mathbf{H}^{2}\right) \times$ $C^{0}\left(H^{1}\right)$, even if the convergence order in space is limited to one, due to the pressure stabilizing term), contains as a sub-product the asymptotic energy balance of the STAB approximation (3.2): The total energy balance is asymptotically maintained in such a way that the sub-grid energy due to stabilizing terms asymptotically vanish (see [19], Section 3.4).

This is not the case if we consider the natural minimal regularity of the continuous solution: Indeed, due to the low regularity of the weak solution, we can just prove an energy inequality, due to the dissipative nature of the STAB approximation (3.2), by using that the sub-grid stabilizing energy terms are positive (cf. [18]).

\section{$5 \quad$ Numerical studies}

Numerical studies with the LPS method (3.2) were performed on the one hand to support the theoretical convergence order predicted by the numerical analysis and stated in 
Theorem 4.6. To this end, Example 5.1 considers a 3D unsteady Beltrami flow in laminar regimes which possesses an analytical solution. On the other hand, the performance of the proposed method is studied at a high Reynolds number flow. In Example 5.2, simulations of a $2 \mathrm{D}$ mixing layer evolving in time at Reynolds number $\mathrm{Re}=10^{4}$ are presented and the obtained results are compared with results from the literature.

\subsection{Laminar regime: Beltrami flow (3D)}

This test is aimed to check the convergence order stated in Theorem 4.6 for the scheme (3.2) applied to the computation of the 3D Beltrami flow in laminar regimes. This example describes a three-dimensional unsteady flow situation in which all terms in the incompressible Navier-Stokes equations play a crucial role (i.e., there are no degenerating terms), and for which a closed-form analytical solution exists. Although unlikely to be physically realized, it was developed in [27] for benchmarking, testing, and validation of 3D incompressible Navier-Stokes solvers.

Setup for numerical simulations. The problem is defined in $\Omega=(-1,1)^{3}$. Its analytical solution is given by:

$$
\begin{aligned}
u_{1}= & -a\left[e^{a x} \sin (a y \pm d z)+e^{a z} \cos (a x \pm d y)\right] e^{-\nu d^{2} t}, \\
u_{2}= & -a\left[e^{a y} \sin (a z \pm d x)+e^{a x} \cos (a y \pm d z)\right] e^{-\nu d^{2} t}, \\
u_{3}= & -a\left[e^{a z} \sin (a x \pm d y)+e^{a y} \cos (a z \pm d x)\right] e^{-\nu d^{2} t}, \\
p= & -\frac{a^{2}}{2}\left[e^{2 a x}+e^{2 a y}+e^{2 a z}+2 \sin (a x \pm d y) \cos (a z \pm d x) e^{a(y+z)}\right. \\
& +2 \sin (a y \pm d z) \cos (a x \pm d y) e^{a(z+x)} \\
& \left.+2 \sin (a z \pm d x) \cos (a y \pm d z) e^{a(x+y)}\right] e^{-2 \nu d^{2} t},
\end{aligned}
$$

where $a$ and $d$ are parameters defining a family of solutions. In our simulations, they were fixed to be $a=\pi / 4$ and $d=\pi / 2$, resulting in initial velocities ranging from 1.59 to -3.31 (cf. [27]). These velocity fields are generated by eigenfunctions of the curl operator ( $c f$. [44]) in such a way that the unsteady term balances the viscous term in the momentum equation, the velocity is divergence-free, and the convective term can be expressed as the gradient of a scalar function (i.e., the negative of the pressure). The sign + was taken in formulas (5.1)-(5.4) to perform the presented numerical simulations, whereas the sign gave similar results.

Following [28, 29], two different flow regimes were considered, a diffusion-dominated flow with $\nu=1$ and a convection-dominated flow with $\nu=10^{-3}$. The Reynolds numbers based on the chosen viscosity, the length of the domain, and the maximum initial velocity (in modulus) were $R e=6.62$ and $R e=6620$, respectively. The initial flow state was the same for both the diffusion- and the convection-dominated flow regime, since the viscosity has no effect at $t=0$. Depictions of the initial velocity and pressure fields can be found in [29].

Due to the balance of the left-hand side terms in the momentum equation, there are no body forces in this problem, so that $\boldsymbol{f}=\mathbf{0}$ in (3.2). Dirichlet boundary conditions based on (5.1)-(5.3) were applied on all faces. The following expressions of the stabilization coefficients were used:

$$
\tau_{\nu, K}=\tau_{p, K}=\left(\frac{1}{\Delta t}+\frac{1}{\tau_{1, K}^{n}}\right)^{-1}, \quad \tau_{1, K}^{n}=\left[c_{1} \frac{\nu}{\left(h_{K} / l\right)^{2}}+c_{2} \frac{U_{K}^{n}}{\left(h_{K} / l\right)}\right]^{-1},
$$




$$
\tau_{d, K}=\frac{\left(h_{K} / l\right)^{2}}{c_{1} \tau_{1, K}^{n}},
$$

by adapting the form proposed in [24, 25], designed by asymptotic scaling arguments applied in the framework of stabilized methods. In (5.5)-(5.6), $c_{1}$ and $c_{2}$ are user-chosen positive constants, $l$ is the degree of the polynomial interpolation, and $U_{K}^{n}$ is some local speed on the mesh cell $K$ at the previous time step $n$ (it should be $U_{K}^{n} \in L^{\infty}(K), n=$ $0,1, \ldots, N-1$, to ensure (3.8)). The values of the constants $c_{1}$ and $c_{2}$ were chosen to be $c_{1}=4, c_{2}=\sqrt{c_{1}}=2$ (cf. [23]), and we set $U_{K}^{n}=\left\|\boldsymbol{u}_{h}^{n}\right\|_{\mathbf{L}^{2}(K)} /|K|^{1 / 2}$. Problem (3.2) was implemented in a FreeFem ++ (cf. [33]) code.

The main interest was in testing the convergence order in space, so that uniform meshes with $4^{3}, 5^{3}, \ldots, 8^{3}$ mesh cells and with $\mathbb{P}_{2}$ finite elements were used for both velocity and pressure, and $l=2$ in (5.5)-(5.6). Starting with the initial field given by (5.1)-(5.3), the semi-implicit Euler scheme (3.2) was applied for the temporal discretization with $N=16$ and $N=32$ time steps of length $\Delta t=0.00625$ and $\Delta t=0.003125$, respectively, resulting in the final simulation time $T=0.1$ in both cases. This approach implies a $22 \%$ decay of the initial flow configuration. According to [27], these setup values optimize the spatial and temporal variation while maintaining reasonable execution times. The time steps chosen ensure that the temporal errors are almost negligible compared with the error in space for all spatial meshes considered.

Numerical results. In Figure 1, the "velocity + pressure" error curves following estimate (4.8) for both the diffusion- and convection-dominated case are presented, related to the grid size $h$, here defined as the distance between adjacent nodes per direction of the mesh cell. The expected convergence order is $\mathcal{O}\left(h^{2}\right)$, due to the use of quadratic finite elements in space and the fact that the temporal error is negligible.
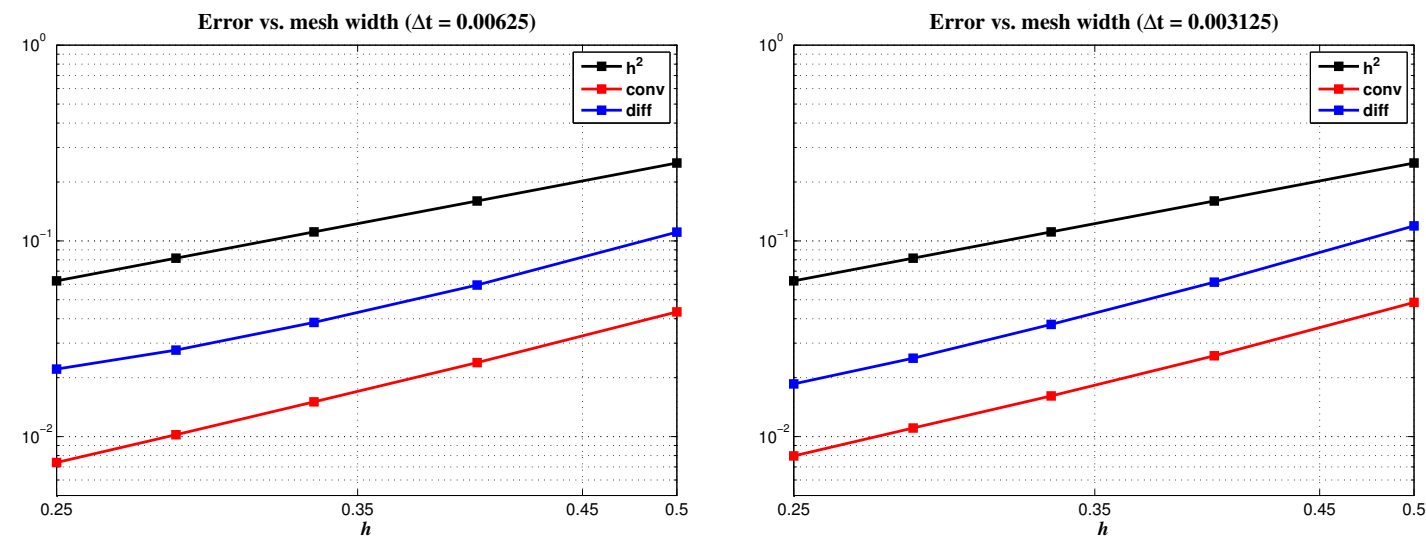

Figure 1:

Example 5.1. Convergence of $\left\|\boldsymbol{u}-\boldsymbol{u}_{h}\right\|_{\ell^{\infty}\left(\mathbf{L}^{2}\right)}+\sqrt{\nu}\left\|D\left(\boldsymbol{u}-\boldsymbol{u}_{h}\right)\right\|_{\ell^{2}\left(\mathbf{L}^{2}\right)}+\left\|\widetilde{P}-P_{h}\right\|_{\ell^{\infty}\left(L^{2}\right)}$ for the diffusion-dominated $\left(\nu=1\right.$, blue) and the convection-dominated $\left(\nu=10^{-3}\right.$, red) case $(\Delta t=0.00625$ on the left and $\Delta t=0.003125$ on the right).

For both cases of this laminar flow (diffusion- and convection-dominated), Figure 1 confirms that the optimal order of convergence, which has to be expected from the error analysis, is achieved. 


\subsection{High Reynolds number regime: Plane mixing layer (2D)}

Numerical results for a plane mixing layer problem evolving in time at relatively high Reynolds number are presented in this section. Mixing layers are encountered in aerodynamics, in the atmosphere or the ocean (e.g., in the wake of mountains, in the Gulf Stream, or in the Mediterranean sea), as well as in the atmospheres of Jupiter and Saturn (at the interface between neighboring zonal jets), confer [40]. Such a flow permits the study of transition to turbulence far from boundaries, and thus the influence of boundaries is removed. The opportunity of considering a two-dimensional problem allows to perform numerical simulations up to a certain level of resolution with the available computer resources. The interaction between two- and three-dimensional turbulence related to this flow has been analyzed in [40].

The plane mixing layer problem has been investigated experimentally, for instance, in [12] for a turbulent regime as well as in [52] at moderate Reynolds number. An extensive review of this type of flows is given in [34]. Numerically, it has been deeply discussed in [40], where a Direct Numerical Simulation (DNS) of a two-dimensional temporal mixing layer problem was performed, applying a second order Finite Difference Method (FDM) at the high resolution of $256^{2}$ grid points with a uniform spacing in each direction. Further numerical studies for this problem, including Large Eddy Simulation (LES), VMS and stabilized models, may be found, e.g., in [9, 13, 30, 31, 36, 37, 46]. The corresponding three-dimensional case has been analyzed numerically, e.g., in [4, 36, 37, 47].

For the setup of our numerical simulations, we chose to follow the guidelines given in [30], where numerical studies of a 2D mixing layer problem for a LES with a three-level VMS finite element method were performed. As a benchmark, we considered the numerical results obtained by the so-called "basic method" in [30], which consists of a Pressure Stabilizing Petrov-Galerkin (PSPG) FEM with an additional grad-div stabilization term.

Setup for numerical simulations. We used a setup similar to the one of [30]. The problem is defined in $\Omega=(0,1)^{2}$. Free-slip boundary conditions were applied at $y=0$ and $y=1$, and periodic boundary conditions were prescribed at $x=0$ and $x=1$. The initial velocity field is given by a hyperbolic tangent basic profile reading:

$$
\boldsymbol{u}_{0}=\left(\begin{array}{c}
U_{\infty} \tanh \left((2 y-1) / \delta_{0}\right) \\
0
\end{array}\right)
$$

where $\delta_{0}$ denotes the initial vorticity thickness, which will be defined below. The initial velocity distribution (5.7) is displayed in Figure 2.

On the initial velocity field (5.7) we superposed a white-noise divergence-free perturbation of small amplitude by means of the streamfunction:

$$
\psi=c_{n} U_{\infty} \exp \left[-\left((y-0.5) / \delta_{0}\right)^{2}\right] \cos (\alpha x),
$$

where $\alpha=2 \pi / \lambda$ is the corresponding wave number with wavelength $\lambda$. This perturbation injects energy into all the longitudinal spatial modes, according to [40], and should reasonably approximate the case of a real mixing layer that is naturally submitted to a residual turbulence having a broadband spectrum.

The mixing layer problem is known to be inviscid unstable. Slight perturbations of the initial condition are amplified by the so-called Kelvin-Helmholtz instabilities. With a linear stability analysis it can be shown that the most amplified mode corresponds to the 


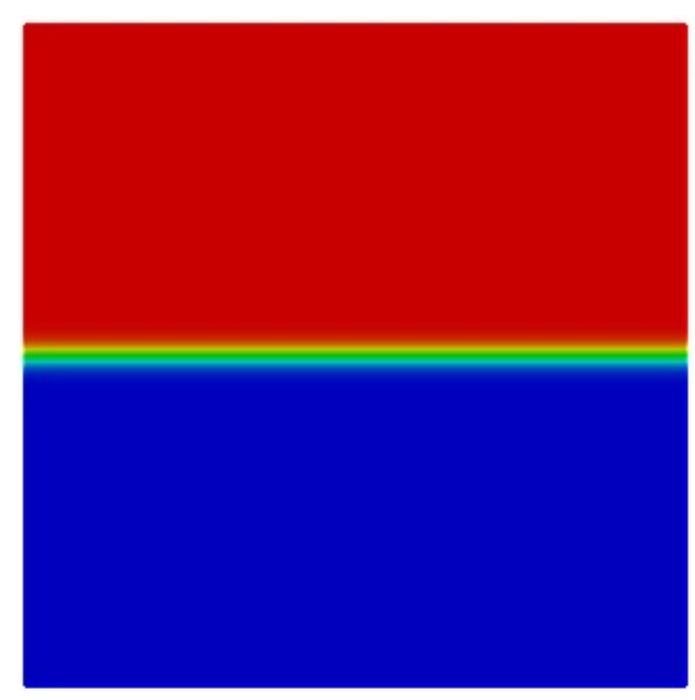

Figure 2:

Example 5.2. First component of the initial velocity (without noise).

most unstable wavelength $\lambda_{a}=7 \delta_{0}$, see [45]. For a domain having extension $L_{x}$ in the stream-wise direction, with $L_{x}=n \lambda_{a}, n \in \mathbb{N}$, the number of primary vortices which are expected to develop in the $x$-direction is equal to $n$. We will present computations with four primary eddies, i.e., $n=4$. Since $L_{x}=1$, we have to choose $\delta_{0}=1 / 28$. We took for the actual perturbation the sum of two waves with wavelengths $1 / 4$ and $1 / 10$ in terms of the domain length, respectively. Consequently, the perturbed initial velocity was given by:

$$
\boldsymbol{u}_{0, \text { Pert }}=\left(\begin{array}{c}
U_{\infty} \tanh \left((2 y-1) / \delta_{0}\right) \\
0
\end{array}\right)+\left(\begin{array}{c}
\partial_{y} \psi \\
-\partial_{x} \psi
\end{array}\right)
$$

with:

$$
\psi=c_{n} U_{\infty} \exp \left(-\left(y / \delta_{0}\right)^{2}\right)(\cos (8 \pi x)+\cos (20 \pi x)) .
$$

The other parameters in the computations were specified to be $U_{\infty}=1$, the scaling factor $c_{n}=10^{-3}$, and the viscosity $\nu^{-1}=28 \cdot 10^{4}$. The Reynolds number associated to this flow is $\operatorname{Re}=U_{\infty} \delta_{0} / \nu=10^{4}$. There are no body forces in this problem, so that $\boldsymbol{f}=\mathbf{0}$ in (3.2).

The stabilization coefficients were chosen as given in (5.5)-(5.6). Three computational grids were used, consisting of uniform $40^{2}, 80^{2}$, and $160^{2}$ partitions of the domain. On these meshes, we consider two-dimensional $\mathbb{P}_{2}$ finite elements for velocities and pressure, so that $l=2$ in (5.5)-(5.6). This choice gives rise to 51200 triangles, 102720 d.o.f. for each scalar variable, and the grid size $h=\sqrt{2} / 160 \approx 8.8388 \cdot 10^{-3}$ for the finest grid.

A time unit $\bar{t}=\delta_{0} / U_{\infty}$ was defined and an equidistant time step of length $\Delta t=0.35 \bar{t}=$ 0.0125 was used. Starting with the perturbed initial velocity field (5.9), the semi-implicit Euler scheme (3.2) was applied as temporal discretization with $N=570$ time steps, resulting in a final simulation time $T=7.125 \approx 200 \bar{t}$. Statistics were collected during the complete simulation time.

Numerical results. For the evaluation of the computational results, we considered the 
vorticity of the flow $\omega=\nabla \times \boldsymbol{u}=\partial_{x} u_{2}-\partial_{y} u_{1}$. The vorticity thickness $\delta\left(t_{n}\right)$ is defined by:

$$
\delta\left(t_{n}\right)=\frac{2 U_{\infty}}{\sup _{y \in[0,1]}\left|\langle\omega\rangle\left(y, t_{n}\right)\right|},
$$

where the numerator indicates the velocity jump across the mixing layer, and $\langle\omega\rangle\left(y, t_{n}\right)$ in the denominator is the integral mean in the periodic direction reading as:

$$
\langle\omega\rangle\left(y, t_{n}\right)=\frac{\int_{0}^{1} \omega\left(\boldsymbol{x}, t_{n}\right) d x}{\int_{0}^{1} d x}=\int_{0}^{1} \omega\left(\boldsymbol{x}, t_{n}\right) d x .
$$

In the computations, this integral was evaluated discretely for all grid lines parallel to the $x$-axis ( $c f$. [37]), and the maximum of these values was employed to obtain $\delta\left(t_{n}\right)$. In the evaluation of the computations, we considered the vorticity thickness scaled by $\delta_{0}$.

Besides the relative vorticity thickness, we also studied the temporal evolution of the total kinetic energy, given by:

$$
E_{\text {kin }}\left(t_{n}\right)=\frac{1}{2} \int_{\Omega}\left|\boldsymbol{u}_{h}^{n}\right|^{2} d \boldsymbol{x} .
$$

In principal, an evolution exhibiting a somehow decaying total amount of kinetic energy has to be expected, since the initial velocity distribution is subject to a non-zero viscosity, and no additional energy input is provided.

Finally, two other aspects of the flow were recorded quantitatively: The mean velocity $\left\langle u_{1}\right\rangle$ and the root-mean-square (r.m.s.) of the velocity $u_{1}$. The mean velocity $\left\langle u_{1}\right\rangle$ at every node was evaluated as a discrete time average over the complete simulation time according to:

$$
\left\langle u_{1}\right\rangle=\frac{1}{N} \sum_{n=1}^{N} u_{1}\left(\boldsymbol{x}, t_{n}\right) .
$$

In addition, these nodal values were spatially averaged along the periodic $x$-direction in order to achieve a final velocity profile along the $y$-direction. The respective r.m.s. value $\sqrt{\left|\left\langle u_{1}^{2}\right\rangle-\left\langle u_{1}\right\rangle^{2}\right|}$ was evaluated during this averaging procedure.

The physical evolution of the flow can be described with the help of Figure 3. These pictures are the result of a simulation using the proposed LPS method (3.2) on the finest grid of $160 \times 160$ mesh cells. They present the evolution of the vorticity $\omega$ through meaningful non-dimensional instants:

- Development of the four primary eddies. Starting with the perturbed initial condition (5.9), the four primary vortices develop, and they can be seen clearly after about 15 time units. This behavior corresponds to the time also observed in [30] using the above mentioned basic method on the same grid, and in [40].

- Pairing of the four primary eddies. The (simultaneous) pairing of the four primary eddies in two secondary eddies takes place at about 35 time units. This behavior compares again to the time observed in [30, 40].

- Pairing of the two secondary eddies. The first pairing is succeeded by a second pairing of the two secondary eddies into one eddy, finished at about 120 time units. This pairing is at a later point in time in comparison with [40] (75 time units), and it is almost comparable with the result from [30] (115 time units). 
- Rotation of the final eddy. After time unit 120, the final eddy rotates at a fixed position. Since this eddy has an elliptic shape, the relative vorticity thickness $\delta / \delta_{0}$ oscillates during this stage, see Figure 4 (black line).
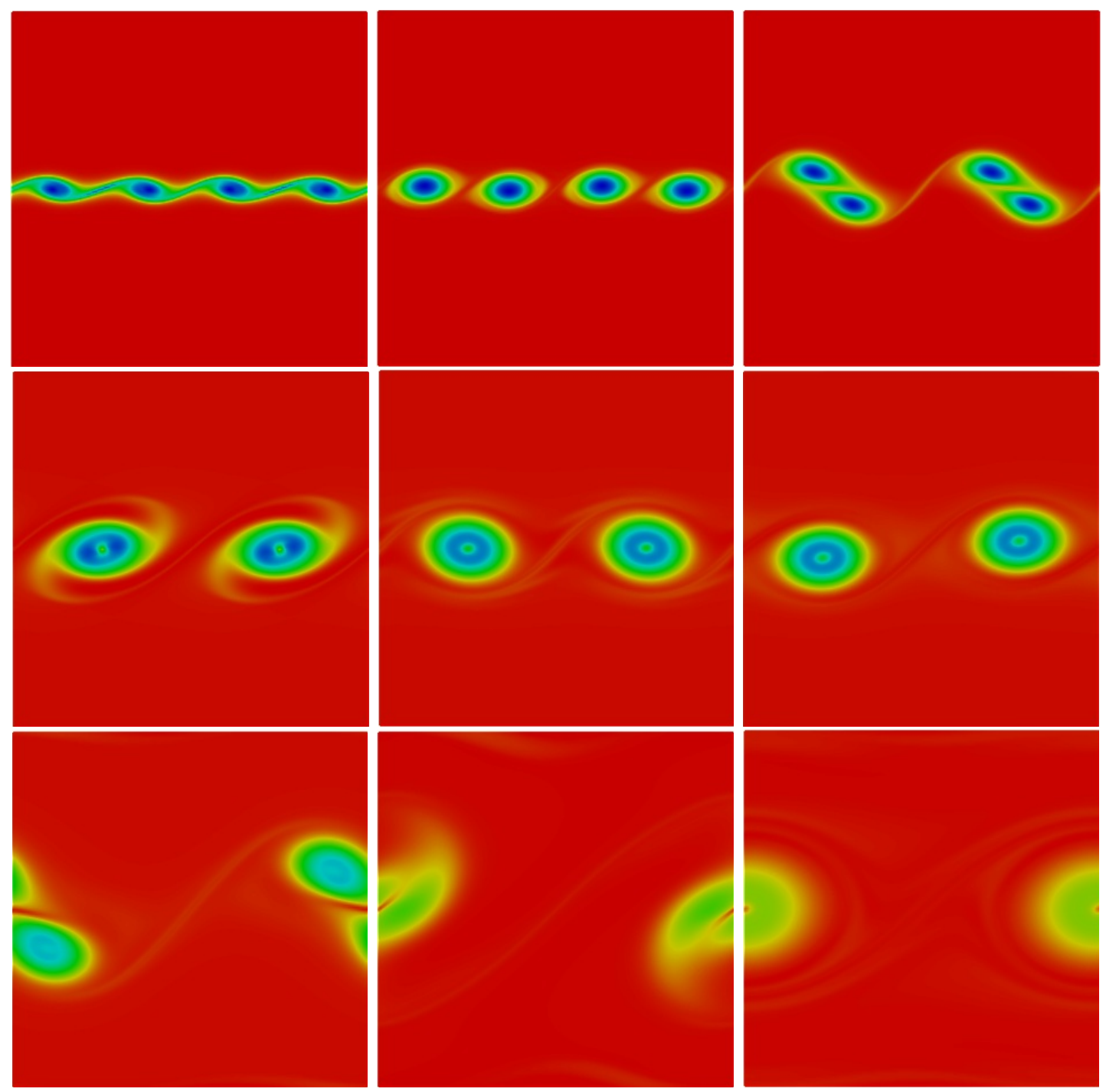

Figure 3:

Example 5.2. Colored vorticity field (blue: intense vorticity, red: irrotational outer flow) at time units 10, 20, 30, 40, 70, 100, 115, 125, 200 (left to right, top to bottom).

The temporal evolution of the relative vorticity thickness $\delta / \delta_{0}$ computed with the proposed LPS method (3.2) on the various grid levels is presented in Figure 4. The main stages of the respective flows, which have been characterized, can be discovered in this picture through the formation of succeeding peaks followed by final oscillations. The maximum values of the vorticity thickness at the first pairing are in between the comparable values in [40] (slightly lower) and [30] (slightly higher), while at the second pairing, the maximal values are slightly higher. The final oscillations are rather contained in amplitude, revealing a slightly elliptic character closer to the results from [40] than from [30], which show larger final oscillations. It can be seen that the vorticity thickness developed differently on different grids. For instance, the coarser the grid, the later the point in time indicating 
the first pairing and, simultaneously, the sooner the second pairing. However, in contrast to the results from [30], the coarse $80 \times 80$ mesh already provides very similar results as the finest $160 \times 160$ grid till the starting point of the second pairing, i.e., till time unit 80 . Moreover, the actual values of the amplitudes of the various peaks are almost identical for all grids considered. Altogether, the grid resolution had a considerable influence on the temporal development of the vorticity thickness, but the values of the various amplitudes stayed unchanged.

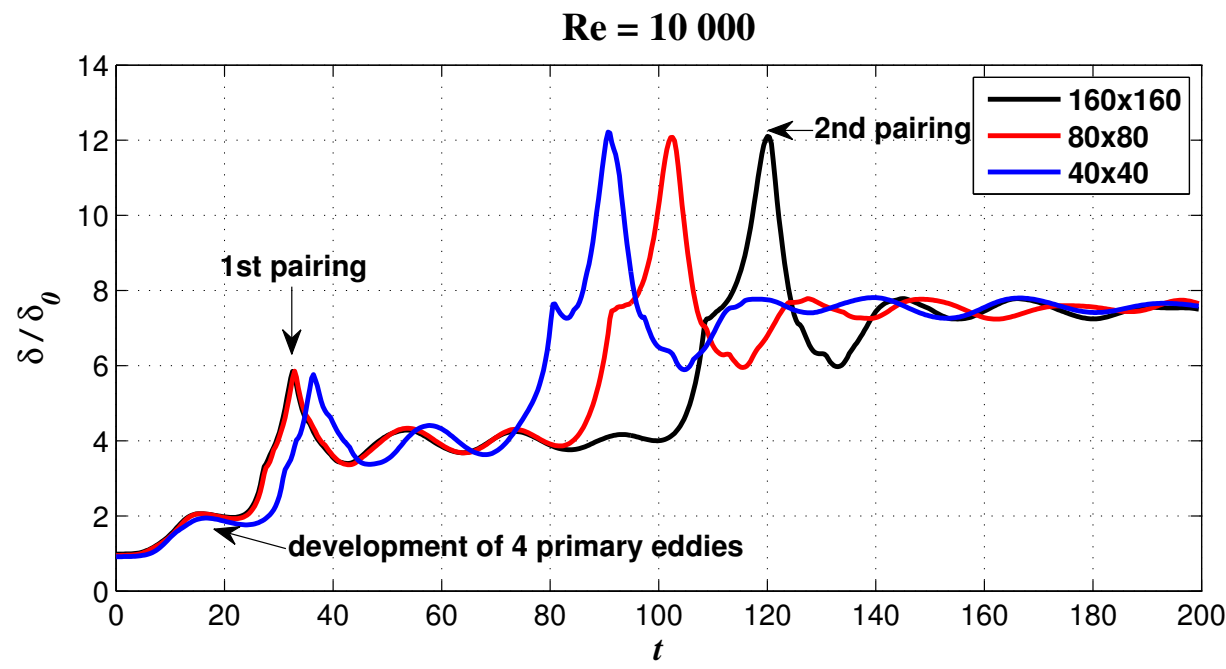

Figure 4:

Example 5.2. Temporal evolution of the vorticity thickness on different meshes.

Concerning the temporal evolution of the total kinetic energy, depicted in Figure 5, it is evident that the lower the resolution level, the higher the overall energy loss, i.e., the more dissipative is the method, as it could be expected. Again, the coarse $80 \times 80$ mesh already gives very similar results as the finest $160 \times 160$ grid till the starting point in time of the second pairing (time unit 80), while a noticeable difference is present between these discretization levels during the complete simulation time in the numerical results in [30].

Figure 6 shows that the mean stream-wise velocity profiles achieved on the various grids are rather close, and in agreement with the results obtained in [30]. The main differences on the various grid levels can be observed in the proximity of the free-slip boundaries. These differences become more pronounced in the curves for the respective r.m.s. values, see also in Figure 6. The lower numerical resolution is clearly reflected in the smaller maximal magnitude of the r.m.s. values. Globally, the maximum values of the r.m.s. stream-wise velocity fluctuations are slightly smaller than the corresponding values in [30].

\section{Summary and conclusions}

In this paper, we have performed a stability and error analysis of the fully discrete unsteady incompressible Navier-Stokes equations discretized with a particular type of LPS method, commonly referred as high-order term-by-term stabilization method. The main contribution of the present paper is the proof of a-priori error estimates for the fully discrete scheme. The analytical results show that for sufficiently regular flow fields an 


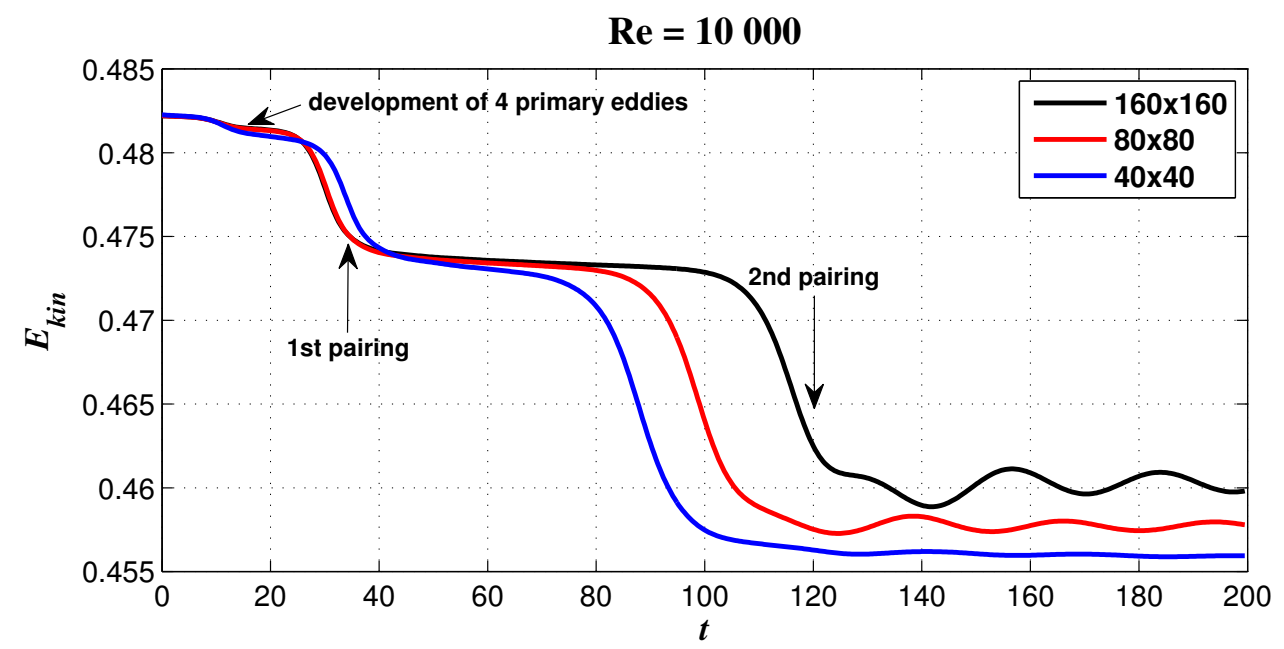

Figure 5:

Example 5.2. Temporal evolution of the total kinetic energy on different meshes.
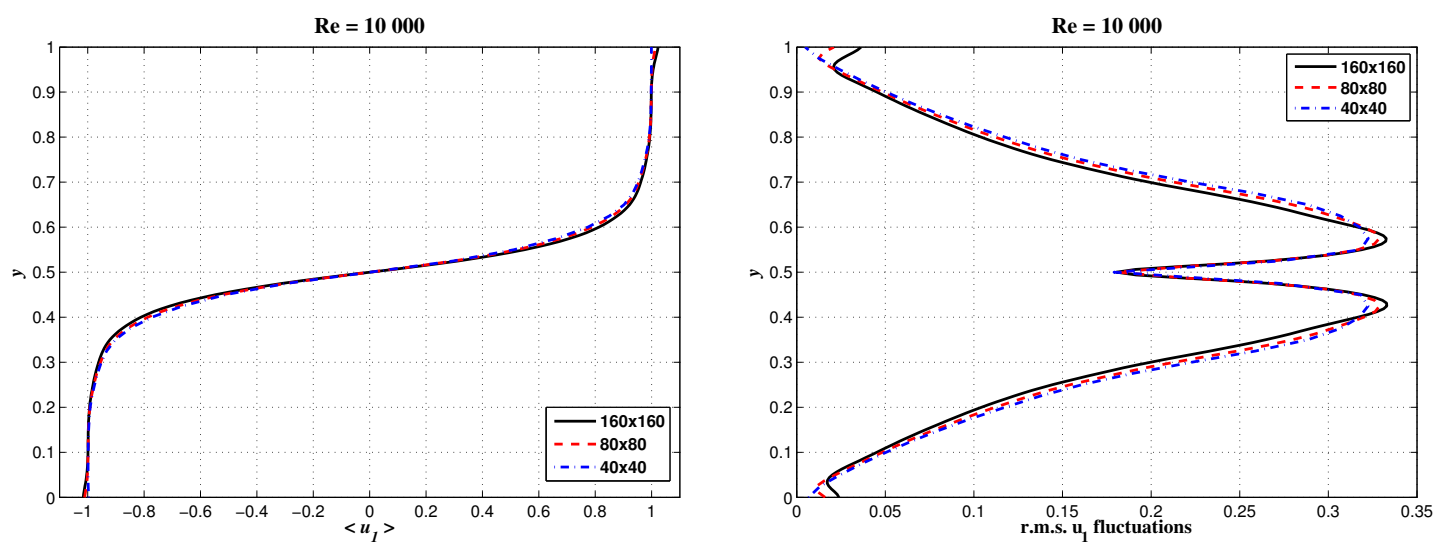

Figure 6:

Example 5.2. Mean stream-wise velocity profiles (left) and r.m.s. stream-wise velocity fluctuations profiles (right) on different meshes.

optimal order of convergence is achieved, which was confirmed by numerical simulations of 3D Beltrami flows. An asymptotic energy balance holds even for less regular flow fields.

Numerical studies of a high Reynolds number plane mixing layer problem indicate that the considered LPS method might be also a useful tool in the challenging simulation of turbulent flows, providing reliable numerical results with a comparatively small computational complexity, which is an extremely important feature in the context of realistic applications in Computational Fluid Dynamics (CFD).

Acknowledgments: The research of Tomás Chacón Rebollo and Samuele Rubino has been partially supported by the Spanish Government project MTM2012-36124-C02-01. 


\section{References}

[1] N. Ahmed, T. Chacón Rebollo, V. John, and S. Rubino. A review of variational multiscale methods for the simulation of turbulent incompressible flows. Arch. Comput. Methods Engrg., Published online:1-50, 2015.

[2] N. Ahmed, G. Matthies, L. Tobiska, and H. Xie. Discontinuous Galerkin time stepping with local projection stabilization for transient convection-diffusion-reaction problems. Comput. Methods Appl. Mech. Engrg., 200(21-22):1747-1756, 2011.

[3] D. Arndt, H. Dallmann, and G. Lube. Local projection FEM stabilization for the time-dependent incompressible Navier-Stokes problem. Numer. Meth. Part. D. E., 31(4):1224-1250, 2015.

[4] E. Balaras, U. Piomelli, and J. M. Wallace. Self-similar states in turbulent mixing layers. J. Fluid Mech., 446:1-24, 2001.

[5] G. R. Barrenechea, V. John, and P. Knobloch. A local projection stabilization finite element method with nonlinear crosswind diffusion for convection-diffusion-reaction equations. ESAIM Math. Model. Numer. Anal., 47(5):1335-1366, 2013.

[6] R. Becker and M. Braack. A finite element pressure gradient stabilization for the Stokes equations based on local projections. Calcolo, 38(4):173-199, 2001.

[7] R. Becker and M. Braack. A two-level stabilization scheme for the Navier-Stokes equations. In Numerical mathematics and advanced applications, pages 123-130. SpringerVerlag, 2004.

[8] C. Bernardi, Y. Maday, and F. Rapetti. Discrétisations variationnelles de problèmes aux limites elliptiques, volume 45 of Mathématiques $\& 3$ Applications. Springer-Verlag, 2004.

[9] B. J. Boersma, M. N. Kooper, F. T. M. Nieuwstadt, and P. Wesseling. Local grid refinement in large-eddy simulations. J. Engrg. Math., 32(2-3):161-175, 1997.

[10] M. Braack and E. Burman. Local projection stabilization for the Oseen problem and its interpretation as a variational multiscale method. SIAM J. Numer. Anal., 43(6):2544-2566, 2006.

[11] M. Braack, E. Burman, V. John, and G. Lube. Stabilized finite element methods for the generalized Oseen problem. Comput. Methods Appl. Mech. Engrg., 196(4-6):853$866,2007$.

[12] G. L. Brown and A. Roshko. On density effects and large structure in turbulent mixing layers. J. Fluid Mech., 64:775-816, 1974.

[13] E. Burman. Robust error estimates for stabilized finite element approximations of the two dimensional Navier-Stokes' equations at high Reynolds number. Comput. Methods Appl. Mech. Engrg., 288:2-23, 2015.

[14] E. Burman and M. A. Fernández. Continuous interior penalty finite element method for the time-dependent Navier-Stokes equations: space discretization and convergence. Numer. Math., 107(1):39-77, 2007. 
[15] T. Chacón Rebollo. A term by term stabilization algorithm for finite element solution of incompressible flow problems. Numer. Math., 79(2):283-319, 1998.

[16] T. Chacón Rebollo, M. Gómez Mármol, V. Girault, and I. Sánchez Muñoz. A high order term-by-term stabilization solver for incompressible flow problems. IMA J. Numer. Anal., 33(3):974-1007, 2013.

[17] T. Chacón Rebollo, M. Gómez Mármol, and M. Restelli. Numerical analysis of penalty stabilized finite element discretizations of evolution Navier-Stokes equation. J. Sci. Comput., 61(1):1-28, 2014.

[18] T. Chacón Rebollo, M. Gómez Mármol, and S. Rubino. Finite element approximation of an unsteady projection-based VMS turbulence model with wall laws. To appear in BAIL 2014 Proc.: Springer Series Lecture Notes in Computational Science and Engineering, 2015.

[19] T. Chacón Rebollo, M. Gómez Mármol, and S. Rubino. Numerical analysis of a finite element projection-based VMS turbulence model with wall laws. Comput. Methods Appl. Mech. Engrg., 285:379-405, 2015.

[20] T. Chacón Rebollo, F. Hecht, M. Gómez Mármol, G. Orzetti, and S. Rubino. Numerical approximation of the Smagorinsky turbulence model applied to the primitive equations of the ocean. Math. Comput. Simulation, 99:54-70, 2014.

[21] T. Chacón Rebollo and R. Lewandowski. Mathematical and numerical foundations of turbulence models and applications. Birkhäuser, 2014.

[22] P. G. Ciarlet. The finite element method for elliptic problems, volume 40 of Classics in Applied Mathematics. SIAM, 2002.

[23] R. Codina. A stabilized finite element method for generalized stationary incompressible flows. Comput. Methods Appl. Mech. Engrg., 190(20-21):2681-2706, 2001.

[24] R. Codina and J. Blasco. Analysis of a stabilized finite element approximation of the transient convection-diffusion-reaction equation using orthogonal subscales. Comput. Vis. Sci., 4(3):167-174, 2002.

[25] R. Codina, J. Principe, O. Guasch, and S. Badia. Time dependent subscales in the stabilized finite element approximation of incompressible flow problems. Comput. Methods Appl. Mech. Engrg., 196(21-24):2413-2430, 2007.

[26] H. Dallmann, D. Arndt, and G. Lube. Local projection stabilization for the Oseen problem. IMA J. Numer. Anal., Published online:1-28, 2015.

[27] C. R. Ethier and D. A. Steinman. Exact fully 3d Navier-Stokes solutions for benchmarking. Internat. J. Numer. Methods Fluids, 19:369-375, 1994.

[28] V. Gravemeier. The variational multiscale method for laminar and turbulent flow. Arch. Comput. Methods Engrg., 13(2):249-324, 2006.

[29] V. Gravemeier, W. A. Wall, and E. Ramm. A three-level finite element method for the instationary incompressible Navier-Stokes equations. Comput. Methods Appl. Mech. Engrg., 193(15-16):1323-1366, 2004. 
[30] V. Gravemeier, W. A. Wall, and E. Ramm. Large eddy simulation of turbulent incompressible flows by a three-level finite element method. Internat. J. Numer. Methods Fluids, 48(10):1067-1099, 2005.

[31] M. Griebel and F. Koster. Adaptive wavelet solvers for the unsteady incompressible Navier-Stokes equations. In Advances in mathematical fluid mechanics (Paseky, 1999), pages 67-118. Springer, Berlin, 2000.

[32] L. He and L. Tobiska. The two-level local projection type stabilization as an enriched one-level approach. Adv. Comput. Math., 36(4):503-523, 2012.

[33] F. Hecht. New development in freefem++. J. Numer. Math., 20(3-4):251-265, 2012.

[34] C. M. Ho and P. Huerre. Perturbed free shear layers. Ann. Rev. Fluid Mech., 16:365424, 1984.

[35] C. O. Horgan. Korn's inequalities and their applications in continuum mechanics. SIAM Rev., 37(4):491-511, 1995.

[36] V. John. Large eddy simulation of turbulent incompressible flows, volume 34 of Lecture Notes in Computational Science and Engineering. Springer-Verlag, Berlin, 2004. Analytical and numerical results for a class of LES models.

[37] V. John. An assessment of two models for the subgrid scale tensor in the rational LES model. J. Comput. Appl. Math., 173(1):57-80, 2005.

[38] P. Knobloch. A generalization of the local projection stabilization for convectiondiffusion-reaction equations. SIAM J. Numer. Anal., 48(2):659-680, 2010.

[39] P. Knobloch and G. Lube. Local projection stabilization for advection-diffusionreaction problems: one-level vs. two-level approach. Appl. Numer. Math., 59(12):2891-2907, 2009.

[40] M. Lesieur, C. Staquet, P. Le Roy, and P. Comte. The mixing layer and its coherence examined from the point of view of two-dimensional turbulence. J. Fluid Mech., 192:511-534, 1988.

[41] G. Matthies, P. Skrzypacz, and L. Tobiska. A unified convergence analysis for local projection stabilisations applied to the Oseen problem. M2AN Math. Model. Numer. Anal., 41(4):713-742, 2007.

[42] G. Matthies, P. Skrzypacz, and L. Tobiska. Stabilization of local projection type applied to convection-diffusion problems with mixed boundary conditions. Electron. Trans. Numer. Anal., 32:90-105, 2008.

[43] G. Matthies and L. Tobiska. Local projection type stabilization applied to inf-sup stable discretizations of the Oseen problem. IMA J. Numer. Anal., 35(1):239-269, 2015 .

[44] D. McLaughlin and O. Pironneau. Some notes on periodic Beltrami fields in Cartesian geometry. J. Math. Phys., 32(3):797-804, 1991.

[45] A. Michalke. On the inviscid instability of the hyperbolic-tangent velocity profile. $J$. Fluid Mech., 19:543-556, 1964. 
[46] S. Nägele and G. Wittum. Large-eddy simulation and multigrid methods. Electron. Trans. Numer. Anal., 15:152-164 (electronic), 2003. Tenth Copper Mountain Conference on Multigrid Methods (Copper Mountain, CO, 2001).

[47] M. M. Rogers and R. D. Moser. Direct simulation of a self-similar turbulent mixing layer. Phys. Fluids, 6(2):903-923, 1994.

[48] H.-G. Roos, M. Stynes, and L. Tobiska. Robust numerical methods for singularly perturbed differential equations. Convection-diffusion-reaction and flow problems, volume 24 of Springer Series in Computational Mathematics. Springer-Verlag, 2nd edition, 2008.

[49] S. Rubino. Numerical modeling of turbulence by Richardson number-based and VMS models. PhD thesis, Univeristy of Seville, 2014.

[50] R. L. Scott and S. Zhang. Finite element interpolation of non-smooth functions satisfying boundary conditions. Math. Comput., 54(190):483-493, 1990.

[51] L. Tobiska and C. Winkel. The two-level local projection type stabilization as an enriched one-level approach. A one-dimensional study. Int. J. Numer. Anal. Model., $7(3): 520-534,2010$.

[52] C. D. Winant and F. K. Browand. Vortex pairing: the mechanism of turbulent mixinglayer growth at moderate Reynolds number. J. Fluid Mech., 63:237-255, 1974. 